ARTICLE

https://doi.org/10.1038/s41467-021-27738-y

\title{
PPAR $\alpha-$ ACOT12 axis is responsible for maintaining cartilage homeostasis through modulating de novo lipogenesis
}

\author{
Sujeong Park ${ }^{1}$, In-Jeoung Baek ${ }^{2}$, Ji Hyun Ryu ${ }^{3}$, Churl-Hong Chun ${ }^{4} \&$ Eun-Jung Jin (iD ${ }^{1 凶}$
}

Here, in Ppara-/- mice, we found that an increased DNL stimulated the cartilage degradation and identified ACOT12 as a key regulatory factor. Suppressed level of ACOT12 was observed in cartilages of OA patient and OA-induced animal. To determine the role and association of ACOT12 in the OA pathogenesis, we generated Acot12 knockout (KO) (Acot12 ${ }^{-/}$) mice using RNA-guided endonuclease. Acot12-/- mice displayed the severe cartilage degradation with the stimulation of matrix MMPs and chondrocyte apoptosis through the accumulation of acetyl CoA. Delivery of acetyl CoA-conjugated chitosan complex into cartilage stimulated DNL and cartilage degradation. Moreover, restoration of ACOT12 into human OA chondrocytes and $O A$-induced mouse cartilage effectively rescued the pathophysiological features of OA by regulating DNL. Taken together, our study suggested ACOT12 as a novel regulatory factor in maintaining cartilage homeostasis and targeting ACOT12 could contribute to developing a new therapeutic strategy for OA.

\footnotetext{
${ }^{1}$ Department of Biological Sciences, College of Natural Sciences, Wonkwang University, Iksan, Chunbuk 54538, Korea. ${ }^{2}$ Asan Institute for Life Sciences, University of Ulsan College of Medicine, Seoul 05505, Republic of Korea. ${ }^{3}$ Department of Carbon Convergence Engineering, Wonkwang University, Iksan, Chunbuk 54538, Korea. ${ }^{4}$ Department of Orthopedic Surgery, Wonkwang University School of Medicine, Iksan, Chunbuk 54538, Korea.

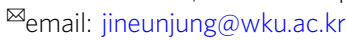


C hondrocytes embedded within the extracellular matrix maintain cartilage homeostasis by stimulating anabolic and catabolic pathways to regulate production, turnover, and degradation of cartilage matrix proteins, such as type II collagen and proteoglycan ${ }^{1}$. During the pathogenesis of osteoarthritis (OA), chondrocytes produce extracellular matrixdegrading proteins such as matrix metalloproteinases (MMPs) as well as pro-inflammatory cytokines such as interleukin (IL)-1 and tumor necrosis factor (TNF) leading to the loss of chondrocyte cellularity and cartilage degeneration ${ }^{2-6}$. OA is a complex disease complicated with several metabolic diseases including dyslipidemia, hyperglycemia, and hypertension ${ }^{7-9}$. Epidemiological studies suggested that obese individuals have significantly increased the incidence of $\mathrm{OA}^{10-12}$. Increased levels of total free fatty acid (FFA) have been reported in OA patients ${ }^{13-15}$ and prior to the appearance of histopathological OA features, accumulation of substantial LD in articular cartilage has been reported $^{9,16,17}$. The elevated level of FFA in chondrocytes may contribute to the accumulation of LDs in articular cartilage ${ }^{18}$. Increased level of saturated fatty acids (SFAs) is closely related to the progression of cartilage degradation ${ }^{19,20}$. High susceptibility to SFA-rich diet is associated with increased OA-like pathophysiological changes ${ }^{20,21}$ and SFA deposits in the cartilage alter the cartilage metabolism to be more prone to damage ${ }^{20}$. Recently, our laboratory also reported that regardless of body mass index (BMI), abnormal lipid accumulation in OA chondrocytes may responsible for the development and progression of $\mathrm{OA}^{22}$. Moreover, regardless of obesity, an increased level of serum cholesterol has been reported to be closely associated with the OA progression ${ }^{14,23}$. Although there is an increasing evidence that excessive lipid deposition (LD) could be associated with $\mathrm{OA}$ pathogenesis, the functional role and regulatory mechanism of LD during OA pathogenesis has not been well established. Therefore, understanding how LDs are regulated and accumulated in chondrocytes and how LD affects the pathogenesis of OA could have a great impact on developing a novel approach or techniques for treating OA.

De novo lipogenesis (DNL) and fatty acid (FA) oxidation, which regulates the breakdown and synthesis of fatty acids, are important determinants of lipid accumulation ${ }^{24,25}$. Lipogenesis is the process that synthesizes the FA chain by combining the acetyl group which adds carbons to a growing FA chain ${ }^{26}$. DNL is a highly regulated pathway and begins with acetyl-CoA as a principal building block for de novo synthesis of fatty acids 26,27 . Acetyl-CoA is a key metabolic intermediate in DNL. Acetyl-CoA, a key indicator of the metabolic state is regulated by adenosine triphosphate (ATP)-citrate lyase (ACLY), acetyl-CoA synthetase 1 (ACSS1), acetyl-CoA synthetase 2 (ACSS2), and acyl-CoA thioesterases (ACOTs). ACLY, ACSS1, and ACSS2 are involved in the production of acetyl-CoA and ACOTs hydrolyze fatty acyl-CoA into free FA and CoA, contributing to pyruvate metabolism and peroxisomal and mitochondrial fatty acyl-CoA oxidation $^{28-32}$. Among them, ACOT12, also known as StarD15 or cytosolic acetyl-CoA hydrolase, is the major cytoplasmic enzyme that preferentially hydrolyzes the thioester bond of acetyl-CoA and generates acetate and $\mathrm{CoA}^{27,33}$. Since ACOT12 may responsible for determining the rate of degradation of cytosolic acetyl-CoA by controlling their dispositions towards oxidation versus complex lipid synthesis, the elucidation of the biological role and function of ACOT12 could provide a molecular basis and mechanism for deregulation of lipid homeostasis involved in the OA pathogenesis and could contribute in developing a new therapeutic approach for OA pathogenesis. In this study, we found that lipid accumulation increased by PPARa deficiency stimulates cartilage degradation through the modulation of ACOT12. In addition, the significant suppression of
ACOT12 in the cartilage of human OA patient and OA animal models, indicating a requisite role of ACOT12 in maintaining articular cartilage homeostasis. We have also demonstrated that ACOT12 deficiency is involved in the LD accumulation of the stimulation of de novo lipogenesis (DNL) through the accumulation of acetyl-CoA, leading to the stimulation of cartilagedegrading enzymes and apoptosis of chondrocyte.

\section{Results}

PPARa is responsible for the accumulation of obeseindependent $\mathrm{LD}$ in the $\mathrm{OA}$ articular chondrocytes. The cartilage of OA patients undergo total knee replacement (TKR) surgery was divided as relatively healthy (non-OA) or severely damaged (OA) region and cartilage degeneration in OA cartilage assessed by Safranin O or Alcian blue staining (Fig. 1a) and Osteoarthritis Research Society International (OARSI) grading ${ }^{34,35}$ (Fig. 1b). We also confirmed the increased expression of matrix metalloproteinase (MMP) 13 in OA cartilage. The LD accumulation in chondrocytes was assessed as staining with BODIPY, a fluorescent probe for neutral lipid or transmission electron microscopy (TEM) analysis (Fig. 1c, d). In this study, to avoid the possible influence or effects of metabolic diseases, sex, and aging in cartilage environment, we studied cartilage tissues in normal BMI $(<25)$ and 50-60-year-old male without hypertension and diabetes mellitus. Consistent with this observation, exposure of interleukin (IL)-1 $\beta$ into human normal articular chondrocyte (HN-AC) cell line and immature murine articular chondrocytes (iMACs) also dramatically induced LD accumulation (Fig. 1e). We also observed a significant increase in the number of BODIPY-positive cells in the cartilage of OA mice induced by destabilization of medial meniscus (DMM) surgery ${ }^{36}$ compared to control cartilage at 8 weeks post surgery (Supplementary Fig. 1). The profile of genes in lipid metabolism using human chondrocytes and iMACs treated with IL-1 $\beta$ (Fig. 1f) as well as GSE dataset (GSE16464; human OA chondrocytes, GSE64394; human OA chondrocytes, GSE104793; IL-1 $\beta$-treated primary mouse articular chondrocytes, GSE104794; HIF-2a-overexpressed primary mouse articular chondrocytes, GSE104795; ZIP8overexpressed primary mouse articular chondrocytes) (Fig. 1g) indicated the involvement of peroxisome proliferator-activated receptor (PPAR) signaling in OA pathogenesis. The expression level of PPARa was significantly decreased in chondrocytes and cartilages of OA patients (Fig. 1h, i) and DMM-induced mouse cartilages at post surgery of 4 - and 6 weeks (Supplementary Fig. 2).

To confirm the involvement of PPARa in the OA pathogenesis, iMACs were treated with fenofibrate, a potent agonists for PPARa, or GW6471, the specific PPARa inhibitor, or introduced by small interference RNA specific to PPARa (siPpara) in the presence or absence of IL-1 $\beta$. Exposure of fenofibrate into iMACs significantly reduced the expression level of matrix metalloproteinase $(M m p)-9, M m p 13$, a disintegrin and metalloproteinase with thrombospondin motifs (Adamts) -4 , and -5 and increased cartilage matrix synthesis with a significant reduction of LD accumulation (Supplementary Fig. 3a-c). In addition, LD accumulation in OA chondrocytes was also significantly reduced by the exposure of fenofibrate (Supplementary Fig. 3d). On the other hand, exposure of GW6471 or introduction of siPpara into IL-1 $\beta$-treated iMACs was stimulated the expression of $M m p 9$, Mmp13, Adatms4, or Adamts5. LD accumulation was also significantly increased by treatment of GW6471 or the introduction of siPpara in IL-1 $\beta$-treated iMACs. Global deletion of PPARa $\left(\right.$ Ppara $\left.^{-/-}\right)$did not change the skeletal development as assessed by alcian blue/alizarin red staining on postnatal day 0 (P0) and the thickness of tibial plateau compared to the cartilage of Ppara ${ }^{+/+}$mice (Supplementary Fig. $4 \mathrm{a}, \mathrm{b}$ ). The presence of sulfated proteoglycans in the culture of $\mathrm{Ppara}^{-/}$iMACs was not 
a

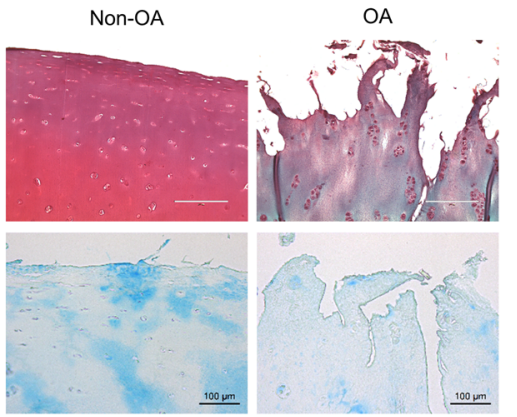

e

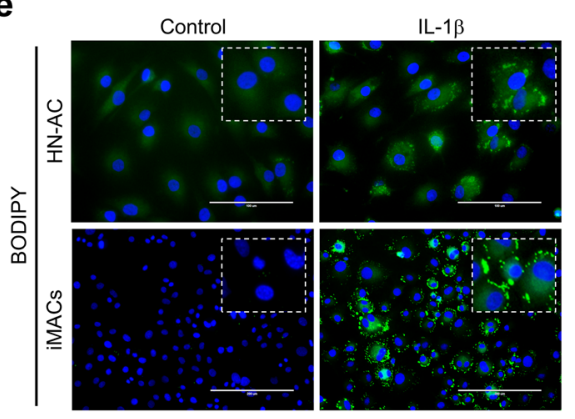

g

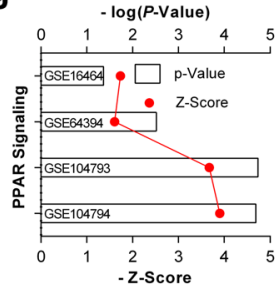

b
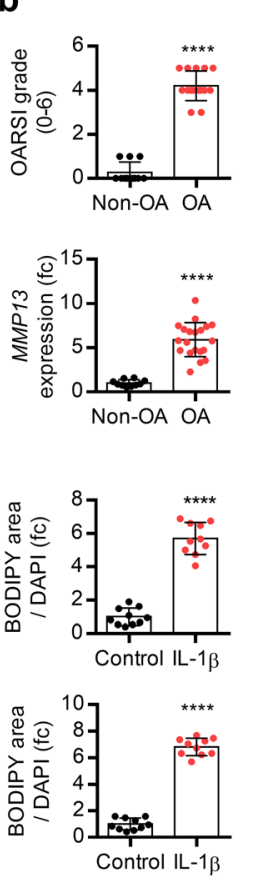

h
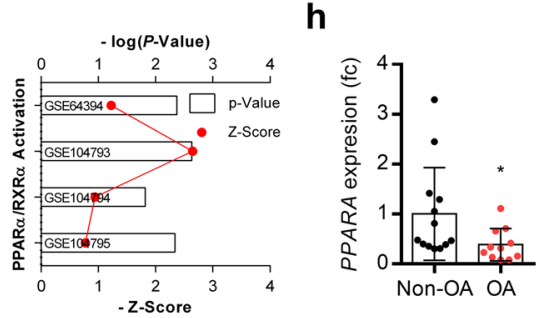

C
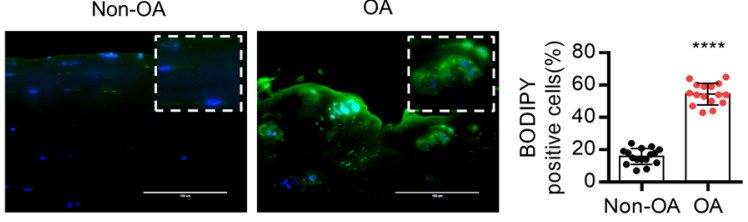

d
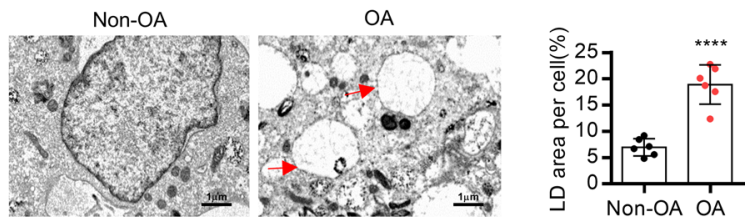

f

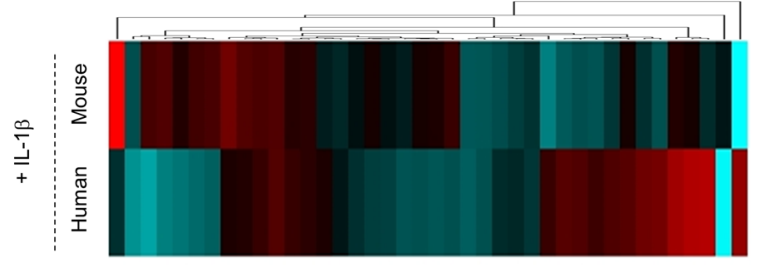

Lipid metabolism involved genes

i

Patient 1

Patient 2

Patient 3
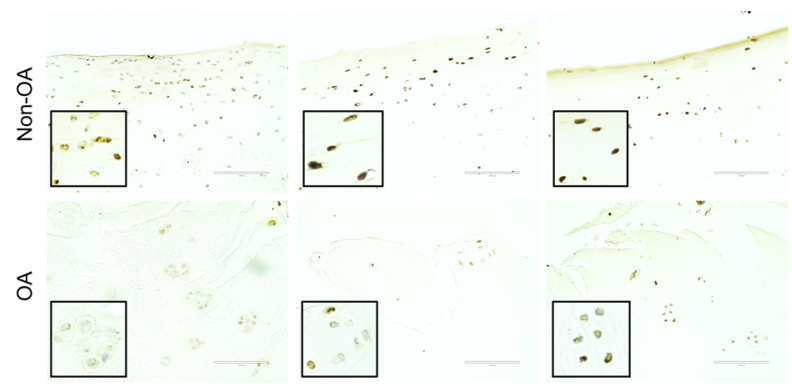

Fig. 1 PPAR is responsible for lipid deposition (LD) during OA pathogenesis. a Safranin $O$ and Alcian blue staining in non-OA and OA cartilages(non$\mathrm{OA}, n=11 ; \mathrm{OA}, n=15$ ). Scale bar, $100 \mu \mathrm{m}$. $\mathbf{b}$ The degree of cartilage degradation is quantified according to OARSI grade. MMP13 mRNA transcription level in non-OA and OA chondrocytes (non-OA, $n=10 ; O A, n=19$ ), $P<0.0001$. c LD is stained by BODPY (green) in intact cartilage isolated from patients (non-OA, $n=16)$ and damaged region $(O A, n=20)$. Scale bar, $200 \mu \mathrm{m}$. $P<0.0001$. d Transmission electron micrographs of non-OA $(n=6)$ and OA $(n=6)$ chondrocytes and LD area were quantified, $P<0.0001$. Red arrow indicates lipid droplet. Scale bar, $1 \mu \mathrm{m}$. e BODIPY staining of immature murine articular chondrocytes (iMACs) and normal human chondrocytes (HN-AC) in the absence or presence of $5 \mathrm{ng} / \mathrm{ml} \mathrm{IL}-1 \beta(n=3), P<0.0001$. $\mathbf{f}$ Expression of

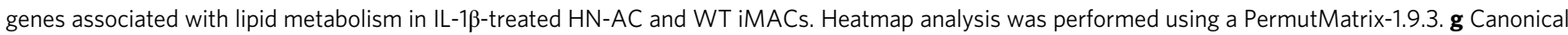
pathway analysis of GSE16464 (human OA chondrocytes), GSE64394 (human OA chondrocytes), GSE104793 (IL-1 $\beta$-treated primary mouse articular chondrocytes), GSE104794 (HIF-2 $\alpha$-overexpressed primary mouse articular chondrocytes), GSE104795 (ZIP8-overexpressed primary mouse articular chondrocytes). $\mathbf{h}$ The expression level of PPARA in non-OA and OA chondrocytes (non-OA, $n=13, \mathrm{OA}, n=11$ ), $P=0.0483$. i Immunohistochemistry of PPARA in non-OA and OA cartilage section of OA patients. The representative region is magnified as dashed line box. Scale bar, $100 \mu \mathrm{m}$. Values are means \pm SD. Unpaired Student's $t$ test $(\mathbf{b}-\mathbf{e}, \mathbf{h})$ was used for statistical analysis. ${ }^{\star} P<0.05$; ${ }^{\star \star \star \star} P<0.0001$.

different from the culture of Ppara ${ }^{+/+}$iMACs (Supplementary Fig. 4c). However, a significant increase in LD accumulation and cartilage degradation and (Fig. 2a) and increased levels of MMP13 and ADAMTS4 (Fig. 2b) were observed in DMMinduced $\mathrm{Ppara}^{-1-}$ cartilage. Restoration of Ppara using lentiviruses containing Ppara (Lenti- Ppara) into joint cavity of Ppara $^{-1-}$ mouse reduced the cartilage degradation, decreased number of BODIPY-positive cells, and decreased expression level of MMP13 and ADAMTS4 in Ppara ${ }^{-/-}$cartilage (Fig. 2c, d).

Decreased level of ACOT12 is responsible for the cartilage degradation by Ppara deficiency. To elucidate the responsible factors in cartilage degradation induced by PPARa deficiency, we analyzed the alteration of expression levels in downstream target genes of PPAR $\alpha$ using Ppara ${ }^{-/-}$iMACs and IL-1 $\beta$-treated Ppara $+/+$ iMACs and identified ACOT12 as a key regulator factor in this (Fig. 3a). The expression level of Acot12 was significantly increased by the introduction of siPpara into iMACs of Ppara ${ }^{+/+}$ mice as well as in iMACs of Ppara ${ }^{-/-}$mice (Fig. 3b, c). Moreover, decreased ACOT12 level and decreased number of ACOT12-positive cells were observed in OA chondrocytes and OA cartilage (Fig. 3d, e). The protein level of ACOT12 in articular chondrocyte was confirmed by western blotting using chondrocyte progenitor cell (CPC) and iMACs (Supplementary Fig. 5a). Consistent with our data, analysis of GSE8077 of rat articular OA chondrocytes also showed the decreased level of ACOT12 in OA chondrocytes (Fig. 3f). Exposure of IL-1 $\beta$ into human normal chondrocytes (HN-AC) or iMACs significantly reduced the expression level of ACOT12 (Fig. 3g). Decreased ACOT12-positive cells were observed in the cartilage of DMMinduced mice (Fig. $3 \mathrm{~h}$ and Supplementary Fig 5b). Introduction of short hairpin RNA against ACOT12 (shAcot12) into HN-AC accumulated free fatty acid and LD with significant increases of 
a

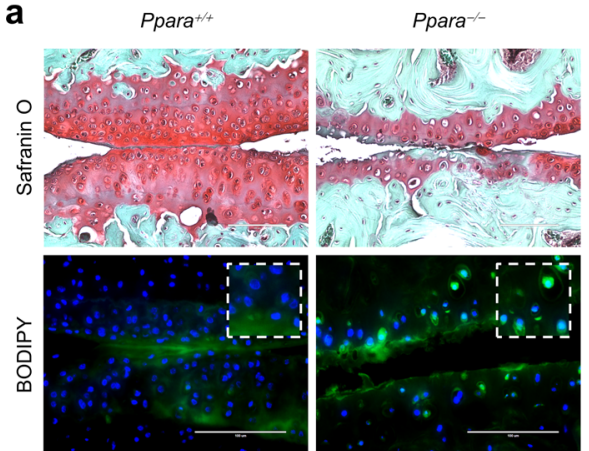

C

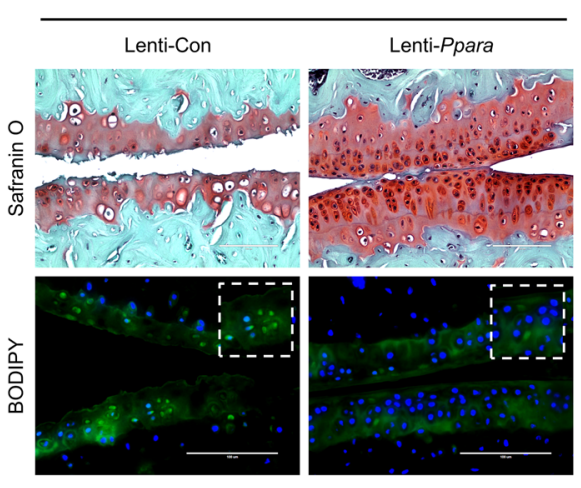

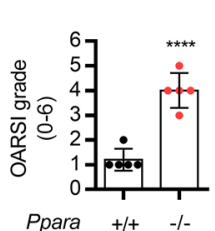
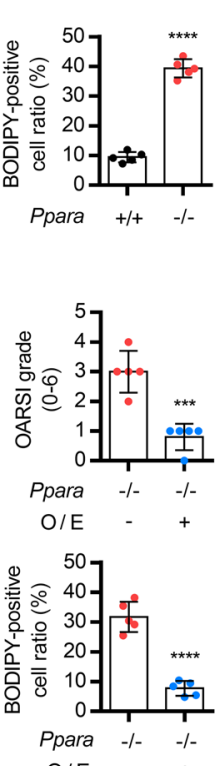

b
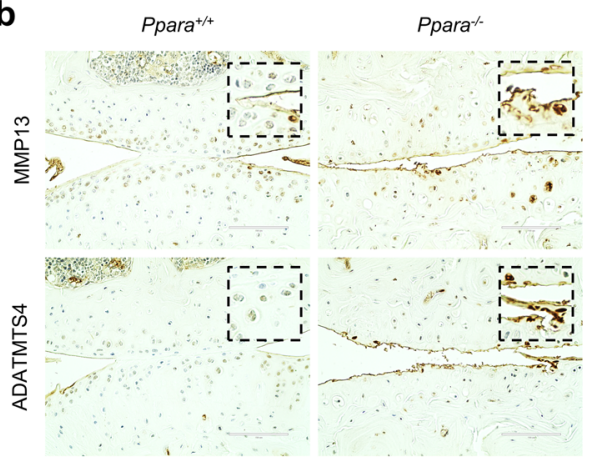

d

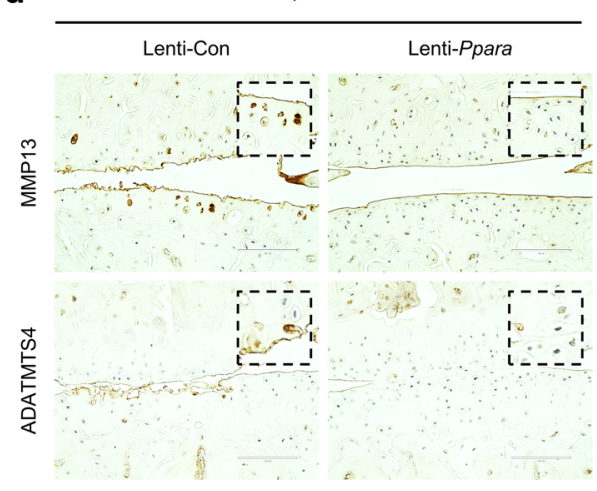

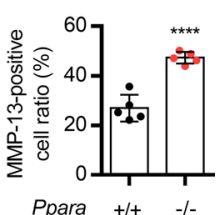
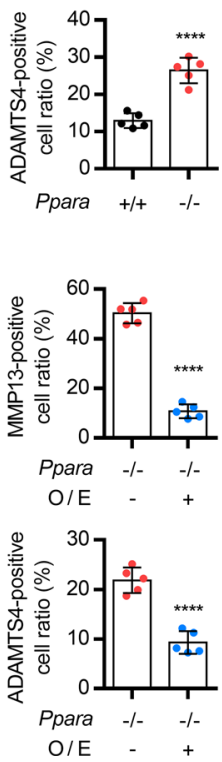

Fig. 2 PPAR $\alpha$ deficiency is involved in OA pathogenesis. a Safranin O or BODIPY staining in the cartilage of Ppara ${ }^{+/+}$and Ppara $/ /-$mice at 8 weeks post surgery. The degree of cartilage degradation is quantified according to OARSI grade and BODIPY-positive cells were indicated by bar-dot plot ( $n=5$ ), $P<0.0001$. Scale bar, $100 \mu \mathrm{m}$. b Immunohistochemistry of MMP13 and ADAMTS4 in Ppara $/+$ and Ppara ${ }^{-/-}$mice at 8 weeks post surgery. Scale bar, $100 \mu \mathrm{m}$. Positive cells of MMP13 and ADAMTS4 were indicated by bar-dot plot $(n=5), P<0.0001$. c Safranin O and BODIPY staining in the cartilage of DMM-induced Ppara ${ }^{+/+}$and Ppara ${ }^{-/-}$treated with Lenti- Ppara. Scale bar, $200 \mu \mathrm{m}$. The OARSI grade, the thickness of the tibia, and BODIPY-positive cells are indicated by graph $(n=5), P=0.0004$ for OARSI grade, $P<0.0001$ for BODIPY. d, Immunostaining of MMP13 and ADAMTS4 in the cartilage of DMM-induced Ppara $/++$ and Ppara $/-$ treated with Lenti- Ppara. Positive cells of MMP13 and ADAMTS4 were indicated by bar-dot plot $(n=5)$, $P<0.0001$. Values are means \pm SD. Unpaired Student's $t$ test $(\mathbf{a}-\mathbf{d})$ was used for statistical analysis. ${ }^{\star \star \star} P<0.001 ;{ }^{\star \star \star \star} P<0.0001$.

MMP13, ADAMTS4, and ADAMTS 5 level (Fig. 3j-1). These data suggest that ACOT12 may act as a key regulator in the cartilage degradation induced by PPARa deficiency.

Acot12 $2^{-/-}$mice display the typical OA characteristics by activating de novo lipogenesis. To investigate the functional role of ACOT12 in vivo, we generated $A \cot 12$ knockout (KO) mice by germline transmission of RNA-guided endonuclease (RGEN)induced mutant Acot12 alleles (Supplementary Fig. 6a). Global deletion of $A \cot 12$ did not alter bone development such as endochondral ossification and postnatal limb length (Supplementary Fig. 6b). Unlike WT $\left(A \cot 12^{+/+}\right)$mice, homozygous KO $\left(\right.$ Acot $\left.12^{-/-}\right)$mice showed signs of spontaneous cartilage destruction at 12 months of age, not at 3 months of age compared to wild-type $\left(A \cot 12^{+/+}\right)$mice (Supplementary Fig. 6c).

Decreased level of cartilage matrix, increase level of lipid peroxidation (Fig. 4a), stimulation of apoptotic cell death (Fig. 4b), decreased level of Acan and Col2a1, increased level of Mmp13, Adamts4 and -5 (Fig. 4c) were observed in Acot12-/iMACs compared to $A \cot 12^{+/+}$iMACs. Severe cartilage destruction and increased number of BODIPY-positive cells were observed in DMM-induced Acot $12^{-/}$mice at 8 weeks of post surgery compared to sham-operated mice (Fig. 4d). MMP13- and ADAMTS4- and TUNEL-positive cells were also significantly increased in DMM-induced Acot $12^{-/-}$cartilage (Fig. 4e).

Increased number of BODIPY-positive cells (Fig. 5a) and increased level of free fatty acid (Fig. 5b) were observed in
IL-1 $\beta$-treated $A \cot 12^{-/-}$iMACs compared to IL- $1 \beta$-treated $A \cot 12^{+/+}$iMACs. Increased level of acetyl-CoA was observed in Acot 12-/- iMACs (Fig. 5c). Moreover, increased level of genes in de novo lipogenesis (DNL) such as acetyl-Coenzyme A carboxylase alpha (Acaca), fatty acid synthase (Fasn), stearoylCoA desaturase $(S c d) 1$ was also observed in $A \cot 12^{-/-}$iMACs (Fig. 5d). Increased number of FASN- and SCD1-positive cells were observed in $A \cot 12^{-/-}$cartilage (Fig. 5e) as well as OA chondrocyte and cartilage of patients (Supplementary Fig. 7).

To determine whether the recovery of ACOT12 into chondrocyte could prevent cartilage degradation, ACOT12-encoding pcDNA (pcDNA-Acot12) were introduced into Acot12 $+/+$ iMACs or $A \cot 12^{-/-}$iMACs in the presence of IL-1 $\beta$ or ACOT12encoding lentivirus (Lenti-HA-Acot12) were injected into knee joint cavity of $A \cot 12^{-/-}$mouse. With the restoration of ACOT12, a significant reduction in triglyceride (TG), decreased number of BODIPY-positive cells, and increased cartilage matrix were observed both in IL-1 $\beta$-treated $A \cot 12^{+/+}$iMACs or $A \cot 12^{-/-}$ iMACs (Supplementary Figs. 8 and 9). Intra-articular injection of Lenti-HA-A $\cot 12$ into $A \cot 12^{-/-}$mouse joint resulted in a decrease in cartilage degradation with an increase in the number of Acot12-positive cells (Fig. 5f). In addition, the expression level of MMP13, FASN, and SCD1 was also inhibited by intra-articular injection of Lenti-HA-Acot12 in DMM-induced mice (Fig. 5h).

Accumulation of acetyl-CoA by ACOT12 deficiency stimulates DNL during OA pathogenesis. Because articular cartilage does 
a

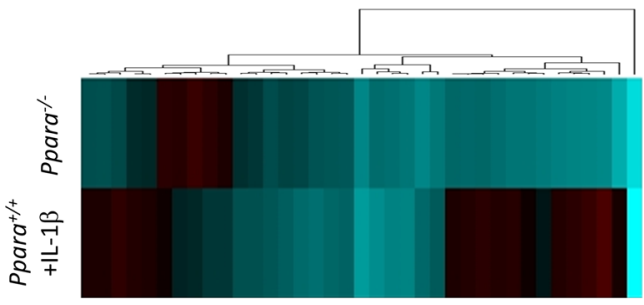

b

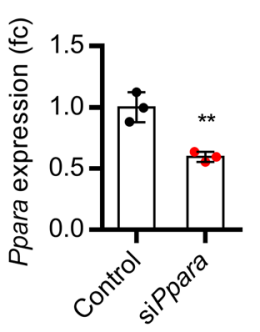

c

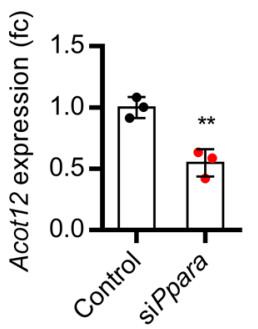

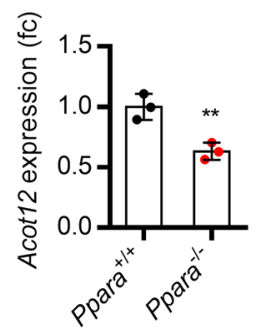

d

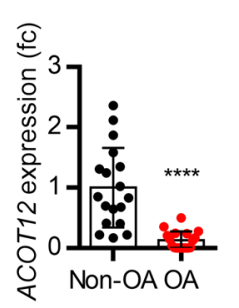

e

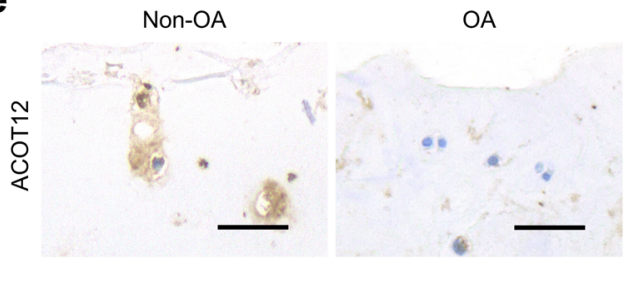

f

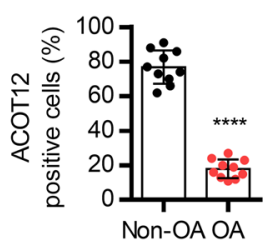

GSE8077

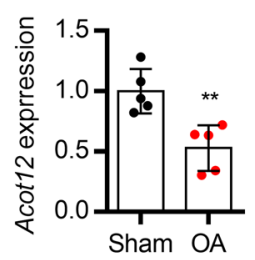

g

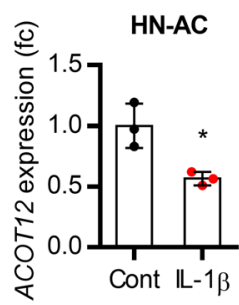

i

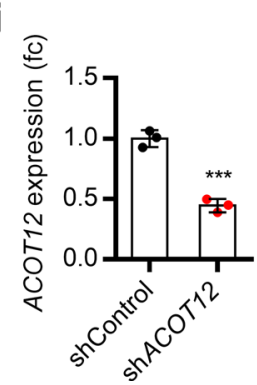

h

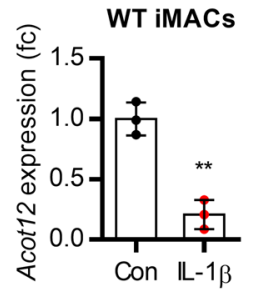

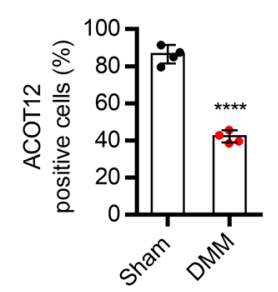

HN-AC
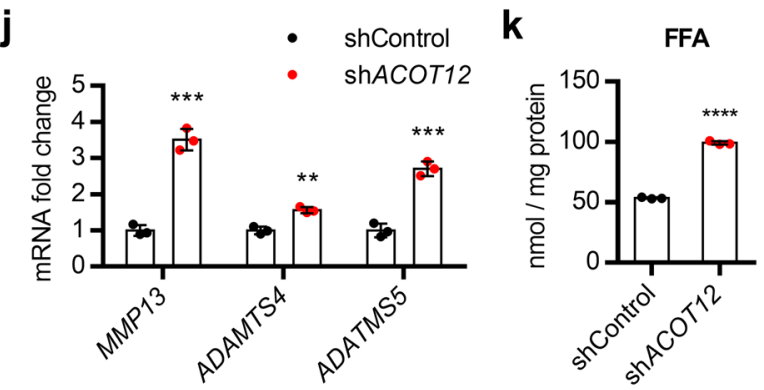

I

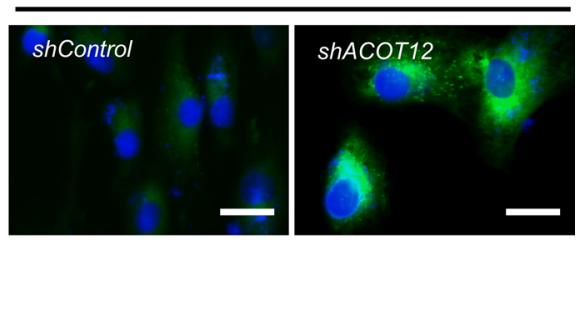

Fig. 3 ACOT12 is a major regulatory factor in OA pathogenesis induced by PPAR $\alpha$ deficiency. a Heatmap analysis of lipid metabolism genes in Ppara $+/+$ and Ppara ${ }^{-/-}$iMACs in the presence of IL-1 1 . Heatmap analysis was performed using a PermutMatrix-1.9.3. b Expression level of Acot12 in iMACs introduce siRNA specific to Ppara (siPpara). Knockdown efficiency of siPpara was confirmed by qRT-PCR $(n=3), P=0.0056$ for Ppara, $P=0.0050$ for Acot12. c Expression level of Acot12 in Ppara ${ }^{+/+}$and Ppara ${ }^{-/-}$iMACs $\left.(n=3), P=0.0077\right)$. d Expression level of ACOT12 in non-OA $(n=18)$ and OA $(n=18)$ chondrocytes $(P<0.0001)$. e Immunohistochemistry of ACOT12 in non-OA and OA cartilage $(P<0.0001)$. Scale bars, $50 \mu$ m. $\mathbf{f}$ Expression of Acot12 in GSE8077 of rat OA articular chondrocytes $(n=5), P=0.0041$. $\mathbf{g}$ Expression level of ACOT12 in HN-AC $(n=3)$ and WT iMACs $(n=3)$, $P=0.0170$ for HN-AC, $P=0.0017$ for WT iMACs. h Immunohistochemistry of ACOT12 in OA-induced mouse cartilage $(n=4), P<0.0001$. Scale bars, $100 \mu \mathrm{m}$. i shACOT12 was introduced into HN-AC $(n=3)$. Knockdown efficiency of shAOCT12 was confirmed by $q R T-P C R(n=3), P=0.0004$. j Expression of Mmp13, Adamts4 and -5 in shACOT12-introduced HN-AC $(n=3), P=0.0002$ for Mmp13, $P=0.0018$ for Adamts4; $P=0.0004$ for Adamts5. k Cellular level of FFA in shACOT12-introduced HN-AC $(n=3), P<0.0001$. I BODIPY staining in shACOT12-introduced HN-AC cells. Scale bars, $20 \mu \mathrm{m}$. Values are means \pm SD. Unpaired Student's $t$ test $(\mathbf{b}-\mathbf{i}, \mathbf{k})$ or multiple $t$ test followed by Holm-Sidak method (j) were used for statistical analysis. ${ }^{\star} P<0.05 ;{ }^{\star \star} P<0.01 ;{ }^{\star \star \star} P<0.001 ;{ }^{\star \star \star \star *} P<0.0001$.

not have blood vessels, nerves, or lymphatics unlike most tissue, chondrocytes mainly depend on anaerobic metabolism using glucose as an important fuel to maintain cartilage homeostasis. Several reports suggested that the glycolytic metabolic pathway converting glucose into lactate when limited amounts of oxygen are available, or pyruvate which then enters the Krebs cycle is upregulated during OA pathogenesis $7,37,38$. In silico analysis of GSE57218, GSE104794 and GSE104795 indicated a significant increase in glycolysis during OA pathogenesis (Supplementary Fig. 10a). Enrichment plot of KEGG and GDEA analysis from RNA-seq data of OA patient (OA vs non-OA) also suggested glycolysis as one of the enriched signaling pathways in OA pathogenesis (Supplementary Fig. 10b). Consistent with these data, Acot $12^{-/-}$iMACs showed activated glycolysis pathway and increased level of glycolysis genes such as hexokinase 2 (HK2), phosphofructokinase (PFKP), phosphoglycerate mutase 2 (PGAM2) and phosphoenolpyruvate carboxykinase $(P C K)$ (Supplementary Fig. 10c, d). Inhibition of glycolysis by 2 -deoxy-D-glucose (2DG) suppressed LD accumulation in the presence of IL- $1 \beta$ without significant differences in chondrocyte apoptosis (Supplementary Fig. 11). These data suggested that LD accumulated in OA chondrocytes may 
a

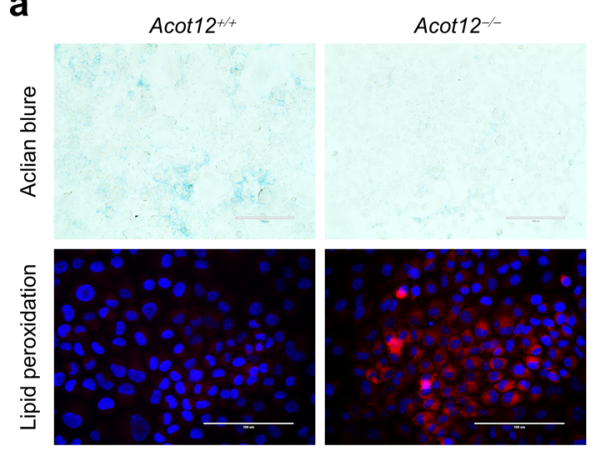

b

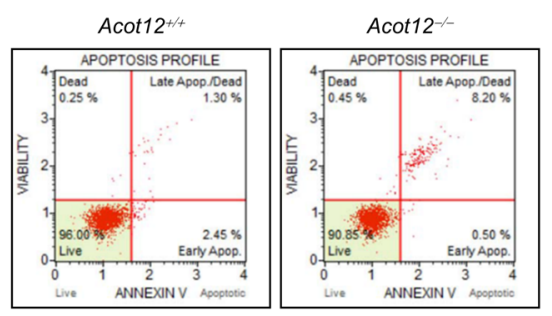

C

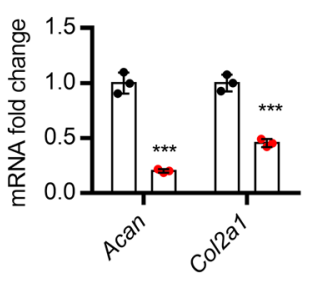

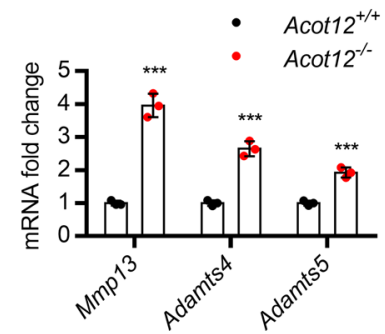

d
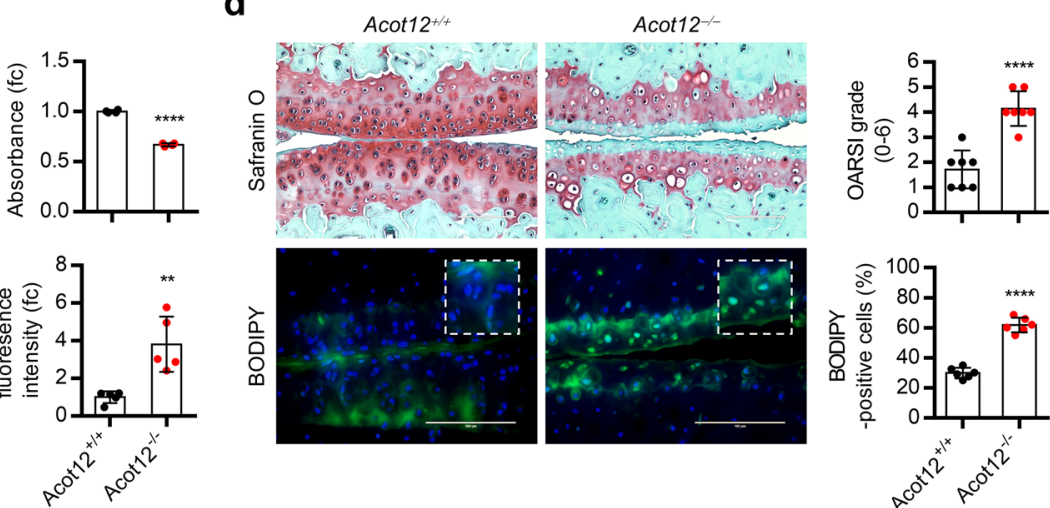

e

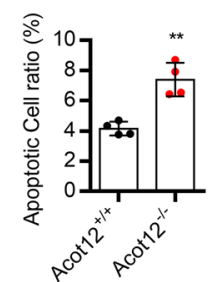

e
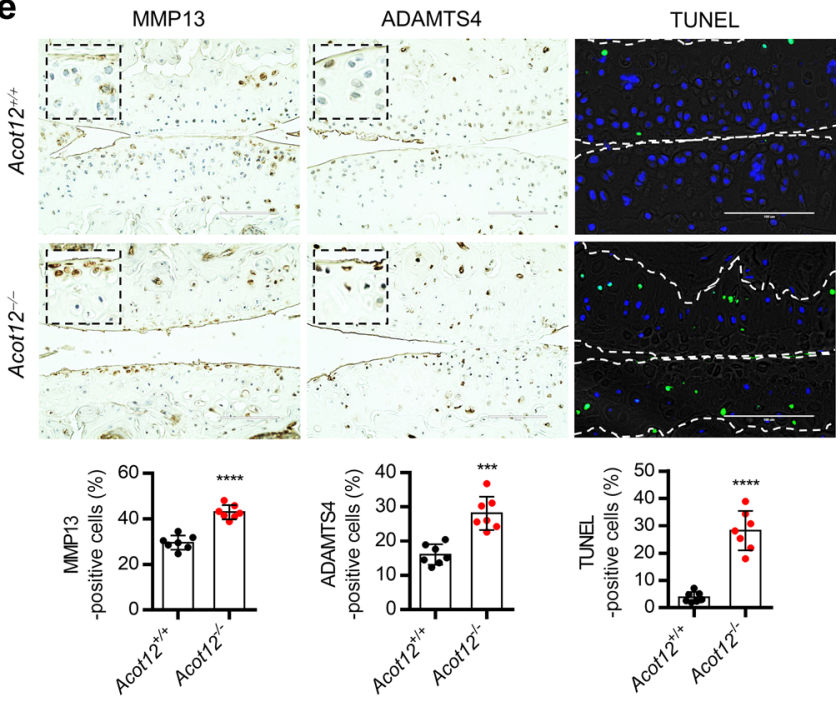

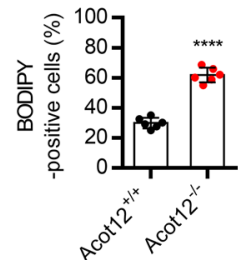

TUNEL

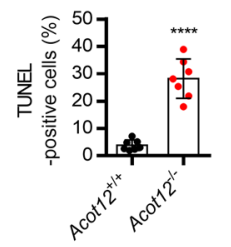

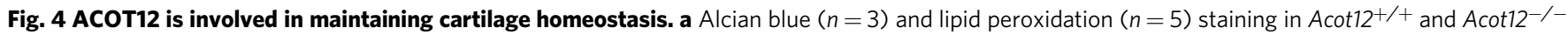
iMACs treated with $5 \mathrm{ng} / \mathrm{ml} \mathrm{IL}-1 \beta, P<0.0001$ for Alcian blue, $P=0.0030$ for lipid peroxidation. b Analysis of apoptotic cell death $(n=4)$ in $A$ cot $12+/+$ or Acot12 $-1-$ iMACs, $P=0.0016$. c Expression level of Acan $(P=0.0001)$, Col2a1 $(P=0.0004), M m p 13(P=0.0002)$, Adamts4 $(P=0.0003)$, and Adatms5 $(P=0.0008)$ in $A \cot 12^{+/+}$and $A \cot 12^{-/-}$iMACs treated with $5 \mathrm{ng} / \mathrm{ml} \mathrm{IL-1 \beta}(n=3)$. d Safranin O and BODIPY staining in cartilage of DMM-induced $A \cot 12^{+/+}$and $A \cot 12^{-/-}$mice at 8 weeks. The degree of cartilage degradation is quantified according to OARSI grade ( $n=7$ ), $P<0.0001$. Scale bar, $100 \mu \mathrm{m}$. BODIPY-positive cells are indicated by bar-dot plot $(n=6), P<0.0001$. e Immunohistochemistry of MMP13 and ADAMTS4 and TUNEL staining in the cartilage section of $A \cot 12^{+/+}$and $A \cot 12^{-/-}$mice $(n=7)$. MMP13 $(P<0.0001)$, ADAMTS4 $(P=0.0001)$, and TUNEL-positive cells $(P<0.0001)$ were indicated by bar-dot plot. Unpaired Student's $t$ test $(\mathbf{a}, \mathbf{b}, \mathbf{d}, \mathbf{e})$ or multiple $t$ test followed by Holm-Sidak method (c) were used for statistical analysis. ${ }^{\star \star} P<0.01,{ }^{\star \star \star} P<0.001,{ }^{\star \star \star \star} P<0.0001$.

derive from glycolysis, not from free FA in the extracellular environment.

In addition, analysis from RNA-seq data of OA patient (OA vs non-OA) showed that expression levels of genes participating in lipogenic pathways such as FA synthesis (ACLY, ACSS2, and ACCL), FA elongation (SCD1, ELOVL5, and ELOVL6), and FA desaturation (FADS2 and FADS3) were significantly increased in OA patient chondrocytes (Supplementary Fig. 12) suggesting that $\mathrm{LD}$ in OA chondrocytes is possibly due to activation of de novo lipogenic pathway (DNL). Since DNL initiated with the conversion of acetyl-CoA into malonyl-CoA, these data suggested that increased acetyl-CoA level by ACOT12 deficiency could activate DNL. Among three major enzymes, ACLY, ACSS2, or ACOT12, involved in the regulation of acetyl-CoA pool ${ }^{33,39,40}$ (Supplementary Fig. 13a), expression levels of ACLY and ACSS2 which are involved in the production of cytosolic acetyl-CoA were significantly increased whereas expression level of ACOT12 which is involved in the hydrolysis of cytosolic acetyl CoA was significantly decreased by IL- $1 \beta$-treated HC-N cells (Supplementary Fig. 13b) and DMM-induced cartilage (Supplementary
Fig. 13c) indicating the accumulation of acetyl-CoA during OA pathogenesis. To investigate the effect of acetyl-CoA accumulation on cartilage homeostasis, iMACs were treated with acetate in a dose-dependent manner. With increasing the concentration of acetate, increased cellular level of acetyl-CoA, FFA, and TG (Fig. 6b), increased expression level of genes in DNL such as Acaca, Fasn, and Scd 1 (Fig. 6c) and in cartilage-degrading enzyme such as Mmp13, Adamts4, and -5 (Fig. 6d) were observed. Furthermore, acetyl-CoA-conjugated chitosan complex (Supplementary Fig. 14a, b) was delivered into articular chondrocyte twice per week by intra-articular injection after DMM surgery 41,42 and delivery efficiency was confirmed by fluorescein isothiocyanate (FITC)-labeled chitosan (Supplementary Fig. 14c). In the cartilage injected with acetyl CoA-conjugated chitosan complex (Chitosan-AcCoA), cartilage degradation as assessed by safranin $\mathrm{O}$ staining and the number of BODIPY- and TUNELpositive cells were significantly increased compared to cartilages injected chitosan alone (Chitosan-Con) and sham-operated cartilage (Supplementary Fig. 15). Increased cartilage degradation and increased number of TUNEL- and MMP13-positive cells 
a
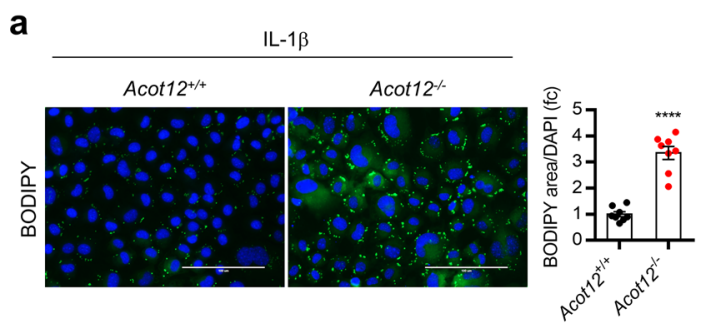

C

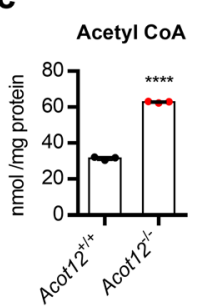

d
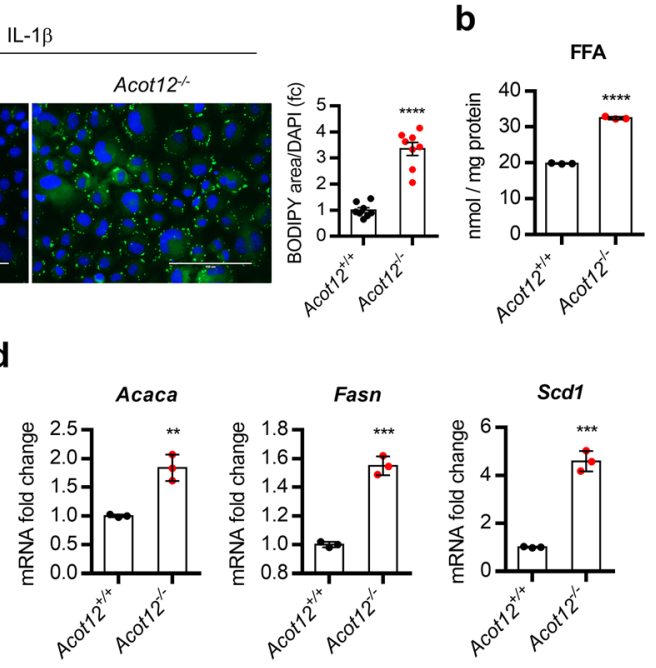

e
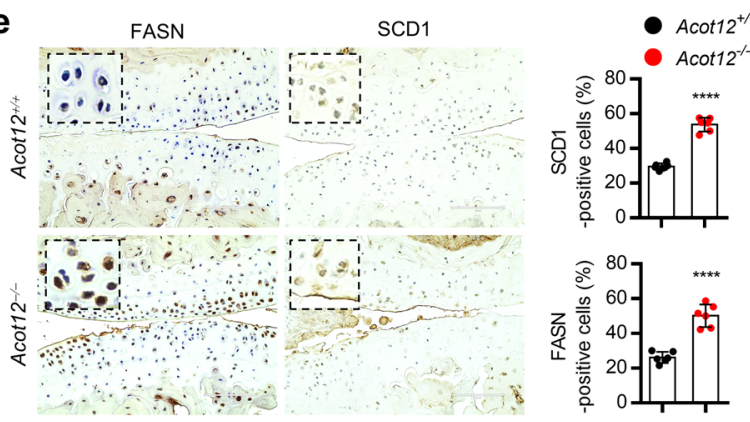
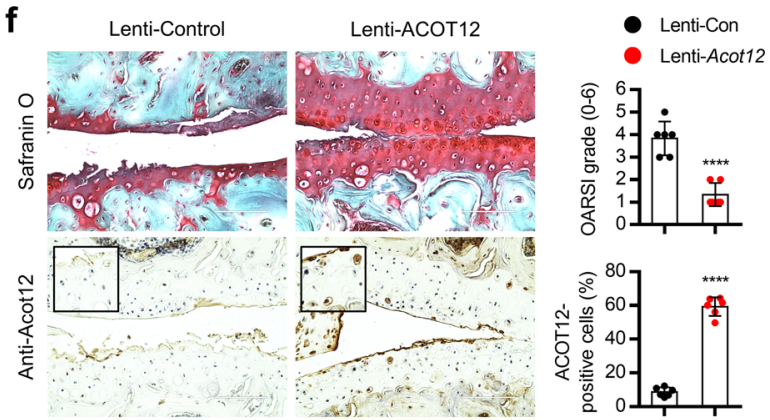

g
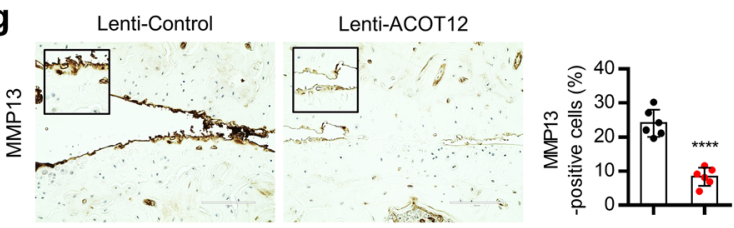

$\mathbf{h}$

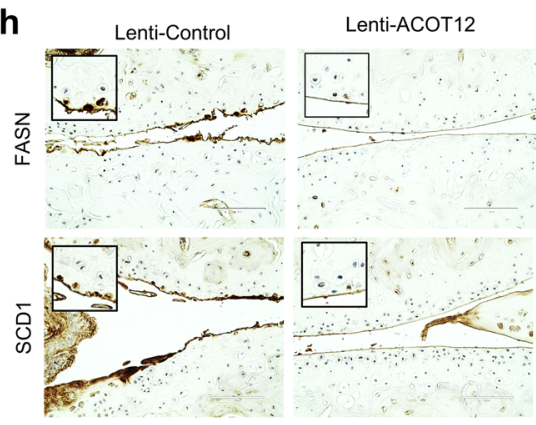

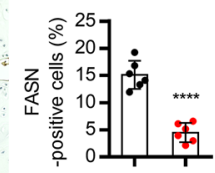

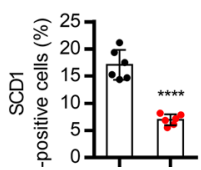

Fig. 5 Acot12-downregulation is involved in OA pathogenesis via DNL. a BODIPY493/503 staining and positive area in $A \cot 12^{+/+}$or $A \cot 12^{-} /-$iMACs in the presence of IL-1 $\beta(n=8) P<0.0001$. Scale bar, $100 \mu \mathrm{m}$. b Cellular level of FFA in Acot12 $/ /+$ or Acot12-/- iMACs $(n=3), P<0.0001)$. c Cellular level of acetyl-CoA in $A \cot 12^{+/+}$or $A \cot 12^{-/-}$iMACs $(n=3), P<0.0001$. d Expression level of Acaca $(P=0.0032)$, Fasn $(P=0.0002)$, and Scd1 $(P=0.0001)$ in $A \cot 12^{+/+}$or $A \cot 12^{-/-}$iMACs $(n=3)$. e Immunohistochemistry and positive cell counting of FASN and SCD1 in $A \cot 12^{+/+}$or $A \cot 12^{-/-}$mice $(n=6)$, $P<0.0001$. Scale bar, $100 \mu \mathrm{m}$. f Safranin O and ACOT12 staining in cartilage of DMM-induced OA mouse with lentiviral ACOT12 $(n=6)$. The degree of cartilage degradation quantified according to OARSI grade and ACOT12-positive cell are indicated by bar-dot plot $(P<0.0001)$. Scale bar, 100 $\mu \mathrm{m}$. $\mathbf{g}$ Immunohistochemistry of MMP13 in cartilage of DMM-induced mouse with lentiviral ACOT12 $(n=6), P<0.0001$. $\mathbf{h}$ Immunohistochemistry of FASN and SCD1 in cartilage of DMM-induced mouse with lentiviral ACOT12 $(n=6), P<0.0001$. Values are means \pm SD. An unpaired Student's $t$ test $(\mathbf{a}-\mathbf{h})$ was used for statistical analysis. ${ }^{\star \star} P<0.01,{ }^{\star \star \star} P<0.001,{ }^{\star \star \star \star} P<0.0001$.

(Supplementary Fig. 15a, b) as well as the increased number of BODIPY-, FASN-, and SCD1-positive cells were observed with the delivery of Chitosan-AcCoA into Acot $12^{+/+}$mice twice per week by intra-articular injection (Fig. 6e, f).

ACOT12 overexpression prevents OA pathogenesis induced by PPARa deficiency. To confirm the involvement of ACOT12 in the OA pathogenesis induced by PPARa deficiency, lentivirus containing HA-tagged ACOT12 (HA-Acot12) were transfected into Ppara $^{-1-}$ iMACs or injected into the cartilage of Ppara ${ }^{-1-}$ mouse. In Ppara $^{-1-}$ iMACs, cartilage matrix was increased and LD accumulation was decreased by introduction of ACOT12 same as shown in the restoration of PPARa (Fig. 7a). Increased level of FFA and acetyl-CoA by IL- $1 \beta$ was also significantly decreased with introduction of ACOT12 as well as the restoration of PPARa (Fig. 7b). Moreover, decreased levels of MMP13, ADAMTS-4, and -5 were observed with introduction of ACOT12 into Ppara-/- iMACs (Supplementary Fig. 16a). Consistent with this, injection of HA-Acot12 into the cartilage of Ppara $^{-1-}$ mice significantly reduced the degradation of cartilage (Fig. 7c and Supplementary Fig. 16b). Increased lipid accumulation and the number of TUNEL-, MMP13-, ADAMTS4-positive cells in $\mathrm{Ppara}^{-1-}$ catilage were significantly decreased by introduction of HA-Acot12 (Fig. 7d, e). These data suggest that
ACOT12 is a major regulatory factor in the OA pathogenesis induced by PPARa deficiency and introduction of exogenous ACOT12 could effectively alleviate cartilage degradation induced by PPARa deficiency.

\section{Discussion}

Fine regulation of metabolism is important for maintaining cartilage homeostasis and impaired metabolism could trigger various pathophysiological situations through stimulation of proinflammatory cytokines and biomechanical stress ${ }^{7,43}$. Thus, metabolic reprogramming in chondrocyte from pathological metabolic state to normal metabolic state by certain enzymes could be one of the possible alternative approaches to OA therapeutic intervention. Several metabolic pathways including glycolysis and fatty acid oxidation are associated with OA pathogenesis ${ }^{7}$. Peroxisome proliferator-activated receptor (PPAR) $\mathrm{s}$, members of the nuclear hormone receptor superfamily in mediating the physiological actions of FA and FA derivatives are known to be involved in the OA pathogenesis ${ }^{44}$. Among three PPAR isoforms, PPAR $\alpha, \operatorname{PPAR} \beta / \delta$, and PPAR $\gamma, \operatorname{PPAR} \gamma$ is the most studied member in OA pathogenesis. PPAR $\gamma 1$, predominantly expressed in human cartilage, is known to have a protective role against $\mathrm{OA}^{45}$. Agonists of PPAR $\gamma$ downregulate inflammatory response and prevent cartilage degradation in $\mathrm{OA}$ 
a

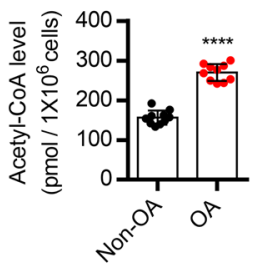

C

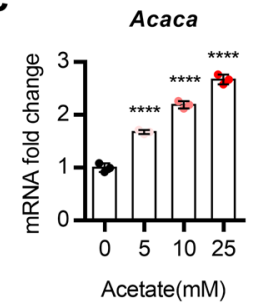

e
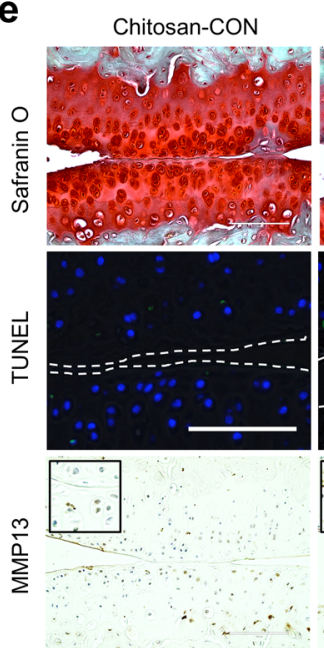

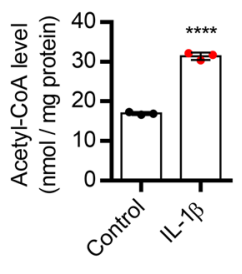

Fasn

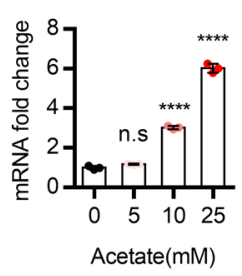

Chitosan-AcCoA
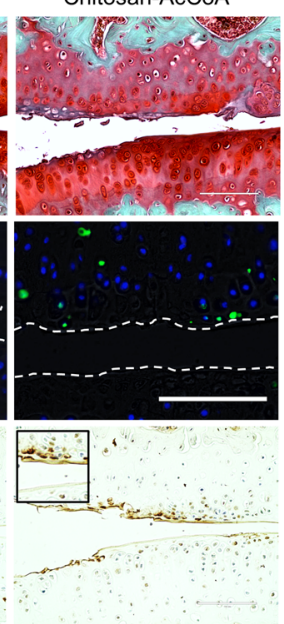

b

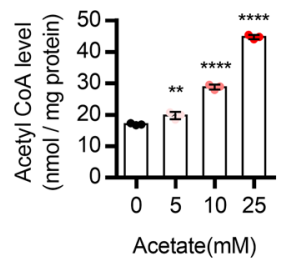

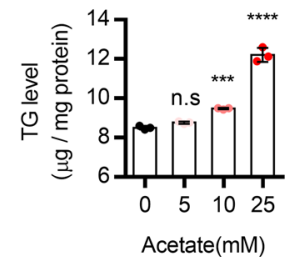

d

Mmp13

Adamts4
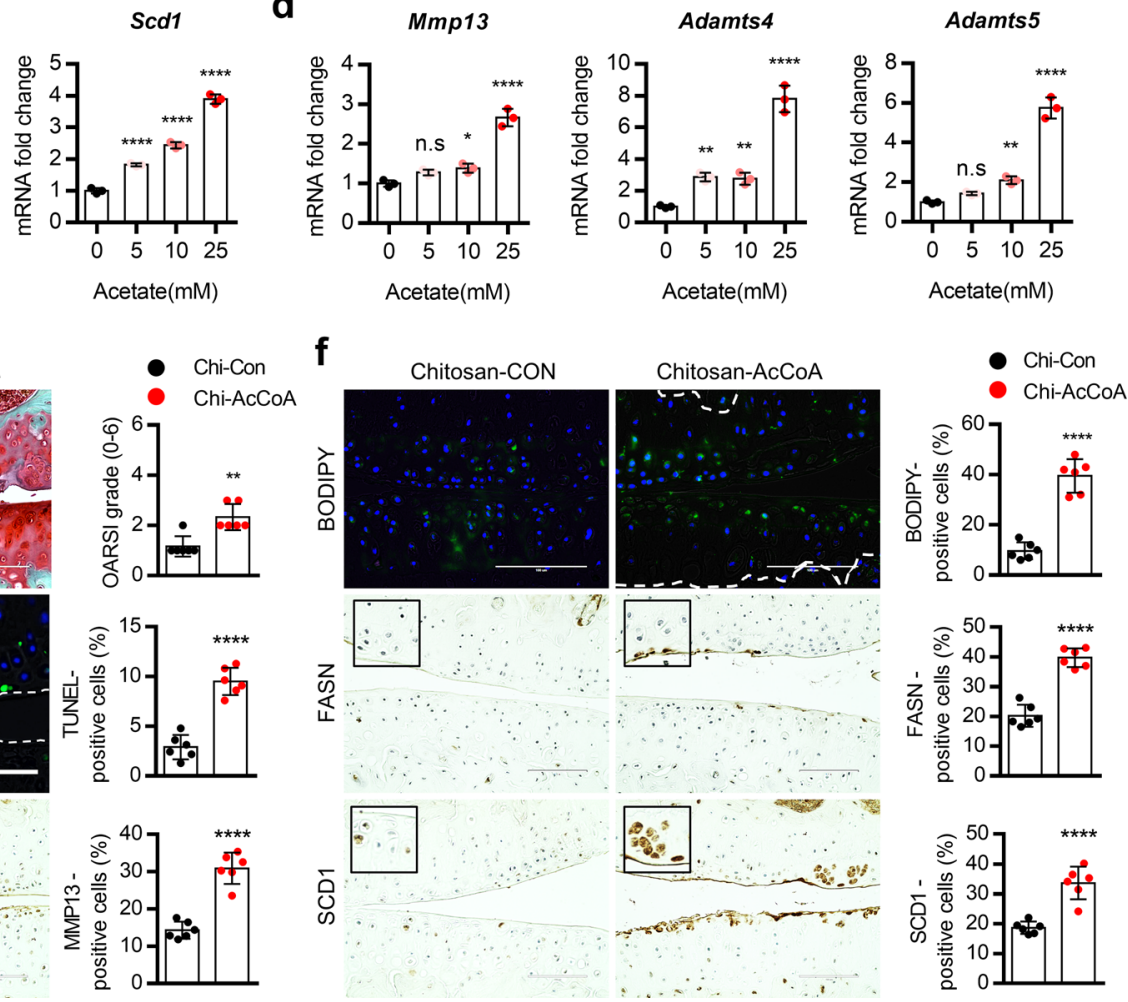

f

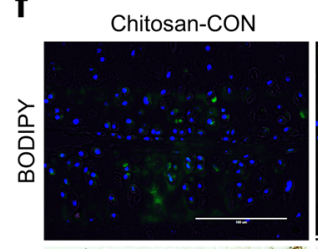

Chitosan-AcCoA
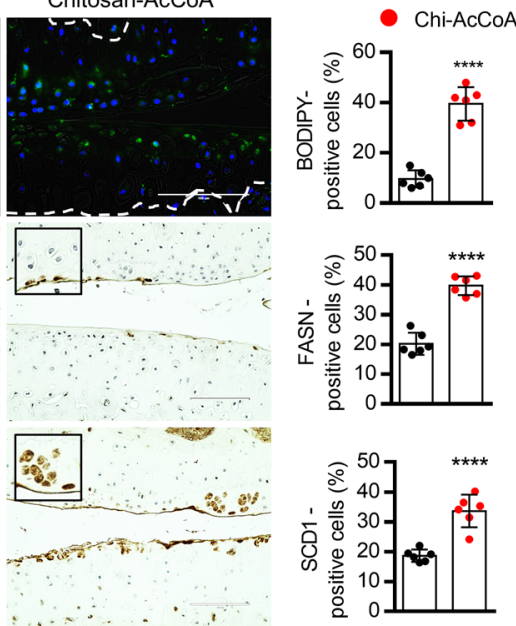

Fig. 6 Acetyl-CoA overload is responsible for stimulation of de novo lipogenesis during OA pathogenesis. a Cellular level of acetyl-CoA in non-OA $(n=10)$ and OA $(n=10)$ chondrocytes (left panel), $P<0.0001$, and iMACs-treated w/wo IL-1 $\beta$ (right panel), $P<0.0001$. b Cellular level of acetyl-CoA ( 0 vs. $5 \mathrm{mM}$ acetate, $P=0.0072,10 \mathrm{mM}$ acetate, $P<0.0001,25 \mathrm{mM}$ acetate, $P<0.0001$ ) FFA ( 0 vs. $5 \mathrm{mM}$ acetate, $P=0.9928,10 \mathrm{mM}$ acetate, $P<0.0001$, $25 \mathrm{mM}$ acetate, $P<0.0001$ ), and TG ( 0 vs. $5 \mathrm{mM}$ acetate, $P=0.2767,10 \mathrm{mM}$ acetate, $P=0.0006,25 \mathrm{mM}$ acetate, $P<0.0001)$ with sodium acetate treatment $(n=3)$. c Expression level of Acaca $(P<0.0001)$, Fasn ( 0 vs. $5 \mathrm{mM}$ acetate, $P=0.3177,10 \mathrm{mM}$ acetate, $P<0.0001,25 \mathrm{mM}$ acetate, $P<0.0001$ for $25 \mathrm{mM})$ and $\operatorname{Scd} 1(P<0.0001)$ with sodium acetate treatment $(n=3)$. d Expression level of Mmp13 ( 0 vs. $5 \mathrm{mM}$ acetate, $P=0.0819,10 \mathrm{mM}$ acetate, $P=0.0199,25 \mathrm{mM}$ acetate, $P<0.0001$ ), Adamts4 ( 0 vs. $5 \mathrm{mM}$ acetate, $P=0.0038,10 \mathrm{mM}$ acetate, $P=0.0053,25 \mathrm{mM}$ acetate, $P<0.0001$ ), and Adamts5 ( 0 vs. $5 \mathrm{mM}$ acetate, $P=0.2391,10 \mathrm{mM}$ acetate, $P=0.0045,25 \mathrm{mM}$ acetate, $P<0.0001$ ) with sodium acetate treatment. e Safranin $O$ staining, TUNEL analysis, and immunohistochemistry of MMP13 in mouse cartilage injected with chitosan $(n=6)$ or chitosan-AcCoA $(n=6)$. The degree of cartilage degradation quantified according to OARSI grade $(P=0.0016)$ and TUNEL- $(P<0.0001)$ and MMP13-positive cell $(P<0.0001)$ are indicated by bar-dot plot. Scale bars, $100 \mu \mathrm{m}$. $\mathbf{f}$ BODIPY staining and immunohistochemistry of FASN and SCD1 in mouse cartilage injected with chitosan or chitosanAcCoA. Scale bars, $100 \mu \mathrm{m}$. BODIPY- $(P<0.0001)$, FASN- $(P<0.0001)$, or SCD1-positive cells $(P<0.0001)$ indicated by bar-dot plot $(n=6)$. Values are means \pm SD. Unpaired Student's $t$ test $(\mathbf{a}, \mathbf{e}, \mathbf{f})$ or one-way ANOVA followed by Dunnett's multiple comparisons test (b-d) were used for statistical analysis. n.s, not significant $P>=0.05 ;{ }^{\star} P<0.05 ;{ }^{\star \star} P<0.01 ;{ }^{\star \star \star} P<0.001 ;{ }^{\star \star \star \star} P<0.0001$.

animal models. Recently several studies have reported that PPARa also plays an important role in cartilage homeostasis ${ }^{46,47}$. PPAR $\alpha$ activation by its agonist, WY14643, decreases inflammatory and cartilage destructive responses in $\mathrm{OA}$ cartilage ${ }^{47}$ suggesting its protective effect on articular cartilage against OA pathogenesis. Here, in this study, we found the protective role of PPARa against cartilage degradation via regulating acetyl-CoA pool through ACOT12 modulation. we also observed that fenofibrate, the agonist for PPARa, reduced the typical characteristics of OA such as increased levels of cartilage-degrading enzymes such as MMP-9, -13 , ADMATS- 4 , and -5 and LD accumulation. Furthermore, we also observed the increase in cartilage degradation and LD accumulation in DMM-induced Ppara ${ }^{-/-}$cartilage.
Accumulating evidences are emerging a key role for metabolites such as ATP, acetyl-CoA, and pyruvate in the development and progression of $\mathrm{OA}^{48-50}$. Among them, acetyl-CoA is known as a central intermediate and a major substrate in many biological responses ${ }^{39,51}$. The level of acetyl-CoA could be competitively regulated by anabolic and catabolic enzymes such as ACC1 and $A C A T 1$ as well as $A C O T 12^{30,52,53}$, which is indispensable for the control of lipid synthesis ${ }^{33}$. ACLY and ACSS2 are the metabolic enzymes responsible for the synthesis of acetyl-CoA from citrate and acetate, respectively ${ }^{40,54,55}$. ACOT12 is the major enzyme known to hydrolyze cytosolic acetyl-CoA and is expressed mainly in hepatocytes and sparsely in the kidney and intestine ${ }^{33,56,57}$. Among these three enzymes, ACLY has been reported as an 
a
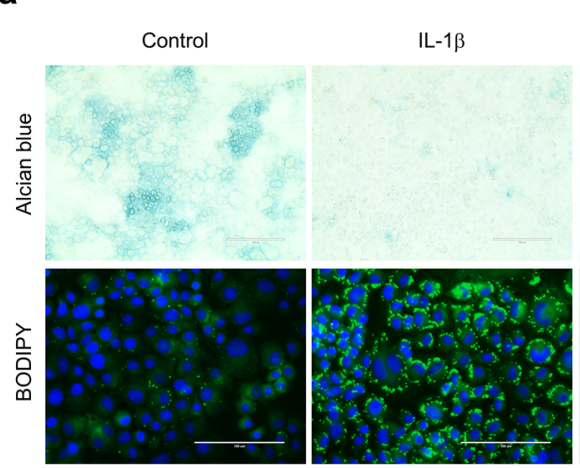

C
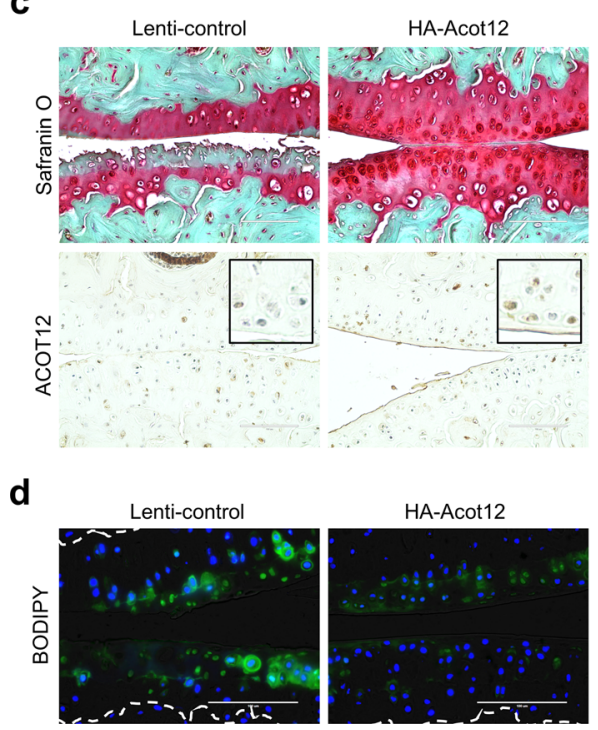
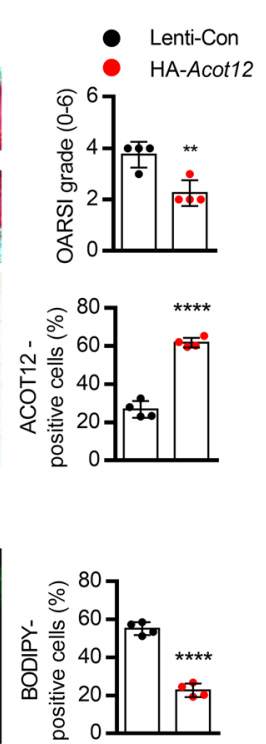

IL-1 $1 \beta$
+ PPAR
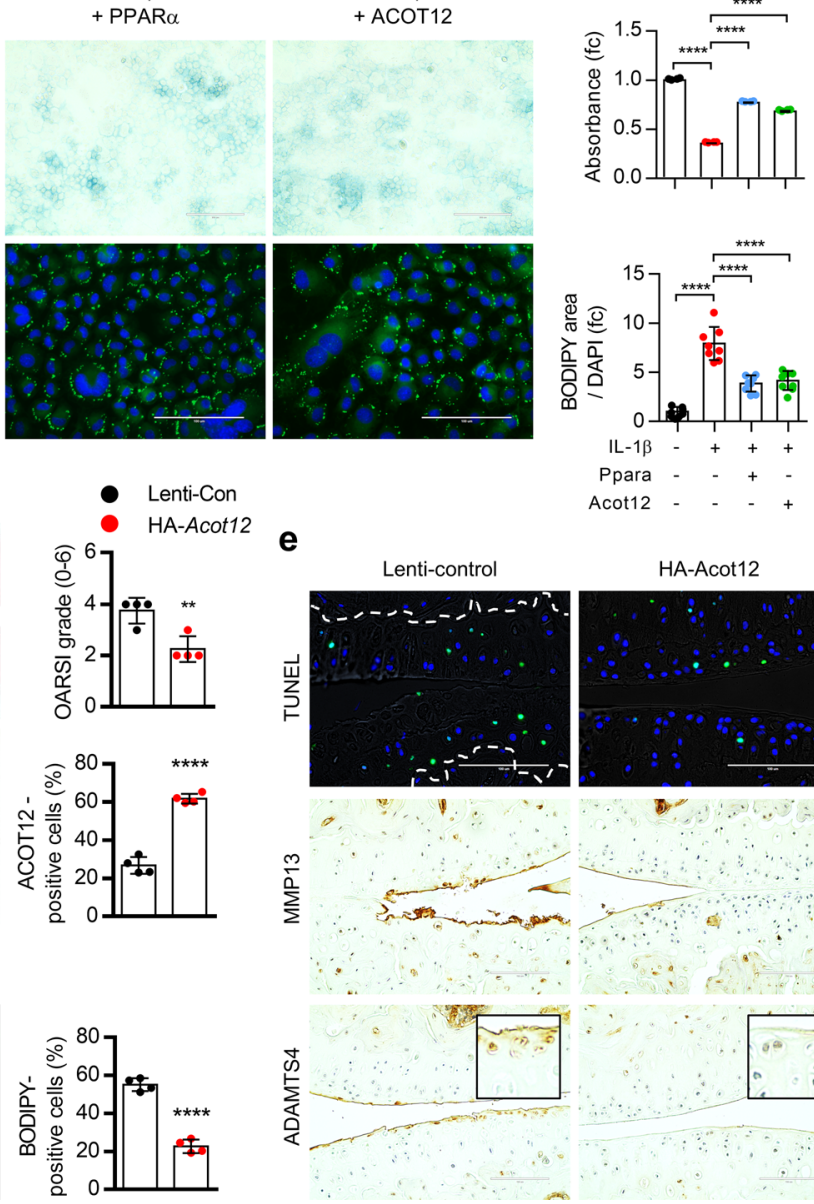

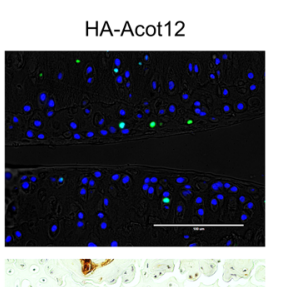

b
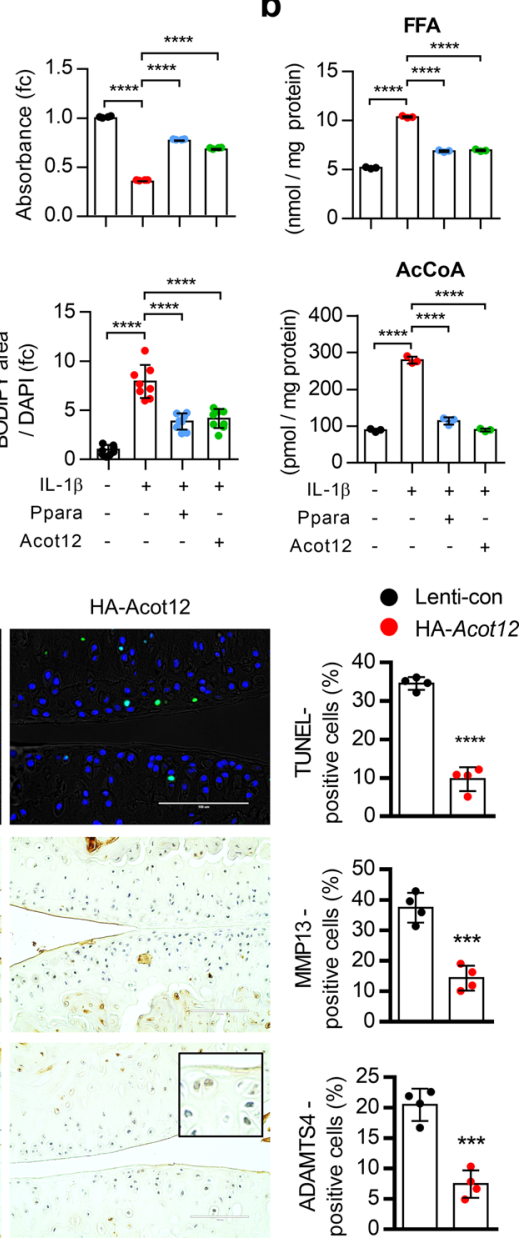

Fig. 7 ACOT12 overexpression alleviates lipid accumulation and OA pathogenesis induced by PPAR $\alpha$ deficiency. a Alcian blue $(n=3)$ and BODIPY $(n=8)$ staining of Ppara ${ }^{-/-}$iMACs introduced by PPAR $\alpha$ or ACOT12 expression vector in the absence or presence of IL-1 $13, P<0.0001$ for Alcian blue, $P<0.0001$ for BODIPY. Scale bars, $100 \mu \mathrm{m}$. b Cellular level of FFA $(P<0.0001)$ and acetyl-CoA $(P<0.0001)$ in Ppara $/-$ iMACs introduced by PPAR $\alpha$ or ACOT12 expression vector in the absence or presence of IL-1 1 . c Safranin O staining and ACOT12 staining in the cartilage of $P p a r a^{-/-}$mice transfected with lentivirus containing HA-tagged Acot12 (HA-Acot12). Scale bars, $100 \mu \mathrm{m}$. OARSI grade $(P=0.0054)$ and ACOT12-positive cells $(P<0.0001)$ indicated by bar-dot plot $(n=4)$. d BODIPY $483 / 503$ staining of in cartilage of Ppara ${ }^{-/-}$mice transfected with HA-ACOT12 $(n=4), P<0.0001$. Subchondral bone indicated with white line. Scale bars, $100 \mu \mathrm{m}$. e TUNEL $(P<0.0001)-$, MMP13 $(P=0.0004)-$, ADAMTS4 $(P=0.0003)$ staining in cartilage of Ppara ${ }^{-/-}$mice transfected with HA-ACOT12 $(n=4)$. Subchondral bone indicated with white line in TUNEL staining. Scale bars, $100 \mu$ m. Values are means \pm SD. Unpaired Student's $t$ test or one-way ANOVA (c-e) followed by Tukey's multiple comparisons test (a, b) were used for statistical analysis. ${ }^{\star \star} P<0.01 ;{ }^{* \star \star} P<0.001 ;{ }^{\star \star \star \star} P<0.0001$.

important regulator for acetyl-CoA accumulation in $\mathrm{OA}$ chondrocytes $^{58}$. In this study, we identified ACOT12 as one of the crucial factors in maintaining cartilage homeostasis by regulating a novel metabolic circuit via acetyl-CoA. We observed a suppressed level of ACOT12 in human OA cartilages and of OAinduced animal cartilages. Germline deletion of Acot12 spontaneously developed articular cartilage lesions without affecting bone development. Cartilage degradation was exacerbated due to a decreased chondrocyte cellularity by activating $M m p-13$ and increasing lipid depositions (LD) in chondrocytes, particularly through acetyl-CoA accumulation in DMM-induced Acot $12^{-/-}$mice.

Then, where do lipid droplets in $A \cot 12^{-/-}$cartilage come from Intracellular lipids can accumulate from an increased lipid influx in blood or stimulation of autophagy or can be synthesized de novo ${ }^{59}$. In this study, we found that de novo lipogenesis (DNL) might be the one key regulatory process for LD accumulation induced by $A \cot 12$ deficiency. In $A \cot 12^{-/-}$cartilage, an increased level of genes in DNL contributing lipid accumulation in chondrocytes was observed and this could lead lipotoxicity and metabolic stress to chondrocytes. Acetyl-CoA is a crucial component in lipid metabolism, as it is a requisite carbon donor in the de novo synthesis of fatty acids and used by acetylCoA carboxylase (ACC) to catalyze the synthesis of malonylCoA. In Acot $12^{-/-}$cartilage, increased level of acetyl-CoA was observed and increased cellular level of acetyl-CoA by treating acetate or delivery of Chitosan-AcCoA into cartilage increased DNL in cartilage and stimulated cartilage degradation by unbalancing between catabolic and anabolic reaction. The recent study also suggests the importance of acetyl-CoA in the OA pathogenesis. Reduced level of acetyl-CoA in cartilage by ACLY deficiency showed a chondroprotective action and resulted less cartilage damage ${ }^{58}$.

Taken together, our study suggested ACOT12 is the main key regulatory factor in PPAR $\alpha$-mediated cartilage homeostasis through regulating acetyl-CoA pool. Increased level of acetyl-CoA by ACOT12 deficiency stimulates DNL pathway and resulted in the stimulation of cartilage degradation. Therefore, targeting 
ACOT12 could be one of the potent therapeutic approaches for controlling cartilage degradation.

\section{Methods \\ Human OA specimens and primary chondrocyte culture. Human articular cartilage specimens were obtained from patients undergoing total knee replacement (TKR) surgery. Specimens from osteoarthritic cartilages were classified as relatively heathy (non-OA) or severe damaged $(\mathrm{OA})$ region. Human articular cartilage specimens were cut into pieces, and some pieces were immediately fixed by $10 \%$ neutral buffered formalin and others used for primary chondrocyte culture. For primary human chondrocyte culture, pieces of each cartilage (OA or non-OA) were digested using $0.06 \%$ collagenase (Sigma) and cultured in high glucose DMEM (Gibco) supplemented with 10\% fetal bovine serum (FBS), $1 \times$ antibiotic-antimycotic (Gibco). The cells were cultured at $37^{\circ} \mathrm{C}$ in a humidified atmosphere of $5 \% \mathrm{CO}_{2}$}

Animals. Wild-type C57BL/6N mice were purchased from Samtako BioKorea Inc. (Osan, Korea). Eight-week-old male C57BL/6N mice used in DMM surgery and 5days-old pups used in iMACs culture. All mice were housed at $23 \pm 1{ }^{\circ} \mathrm{C}$ with 12 -h light/dark cycles and a relative humidity of $50 \pm 5 \%$ with food and water available ad libitum.

Experimental OA and histology of $O A$ cartilage. Experimental OA was induced by destabilization of the medial meniscus (DMM) surgery using 8-weeks-old male mice. Knee joints were analyzed 8 weeks after DMM surgery. Cartilages were processed for histological and biochemical analyses. Briefly, knee joints were fixed in $4 \%$ paraformaldehyde, decalcified in $14 \%$ EDTA ( $\mathrm{pH} 7.4$ ), and embedded in paraffin. The paraffin blocks were sectioned at $5-\mu \mathrm{m}$ thickness and sectioned joints were performed safranin $\mathrm{O}$ staining. The degree of cartilage degradation was scored 0-6 grade using OARSI scoring system and images were acquired by EVOS FL Auto software v.1.7.

Generation of $\boldsymbol{A c o t 1 2}^{-/-}$mice. Global Acot12 knockout mice were generated by germline transmission of an RGEN-induced mutant allele. Acot12 specific guide RNA and Cas9 protein (each $50 \mathrm{ng} / \mathrm{\mu l}$ ) were injected into the cytoplasm of C57BL/ $6 \mathrm{~N}$ mouse eggs and transferred into the pseudo-pregnant foster female mice. The absence of Acot12 in the mutants was confirmed by routine tail DNA genotyping and western blotting. The absence of Acot 12 was confirmed by standard PCR genotyping. Genotyping primers were designed to distinguish $A \cot 12^{+/+}$or Acot $12^{+/-}$or $A \cot 12^{-/-}$alleles: forward: 5'-agccaggacgatggagtcga-3'; reverse: 5'ggtgtccatccacttgagca-3'.

Transmission electron microscopy. Chondrocytes fixed in $2.5 \%$ glutaraldehyde solution were post-fixed with $0.1 \mathrm{M} \mathrm{OsO}_{4}$ and embedded with Epoxy Embedding Medium Kit (Sigma-Aldrich). The epoxy embedded block was ultramicrosectioned using EM UC7 (Leica) and stained with uranyl acetate and lead citrate. The microstructure image was analyzed with $120 \mathrm{kV}$ transmission electron microscope (HITACHI).

Alcian blue staining. Cells were rinsed with PBS and fixed in $4 \%$ paraformaldehyde for $20 \mathrm{~min}$. Fixed cells were stained in $1 \%$ Alcian blue in $0.1 \mathrm{~N} \mathrm{HCl}$ for $24 \mathrm{~h}$ at room temperature, rinsed with $0.1 \mathrm{~N} \mathrm{HCl}$, and captured bright-field image by EVOS FL Auto software v.1.7. Stained Alcian blue was extracted by $6 \mathrm{M}$ guanidine $\mathrm{HCl}$ for $2 \mathrm{~h}$ and measured absorbance at $600 \mathrm{~nm}$.

Whole-mount skeletal staining. Postnatal 0 pups were euthanized and removed skin, visceral organ, eye, adipose tissue, and other excess tissue. Specimens were fixed by $95 \% \mathrm{EtOH}$ for $24 \mathrm{~h}$. Specimens were submerged in alcian blue solution overnight at room temperature and destained in $95 \% \mathrm{EtOH}$. To clear the tissue, solution was replaced by $2 \% \mathrm{KOH}$ solution for $12 \mathrm{~h}$ and replaced with Alizarin red solution for $24 \mathrm{~h}$ at RT. Stained specimens were replaced by $20 \%$ glycerol $/ 1 \% \mathrm{KOH}$, $50 \%$ glycerol $/ 1 \% \mathrm{KOH}$, and $80 \%$ glycerol $/ 1 \% \mathrm{KOH}$ for $24 \mathrm{~h}$ each and transferred to $100 \%$ glycerol.

Biochemical analysis. The cellular level of FFA and acetyl-CoA were measured using Free Fatty Acid Assay Kit (Dogen; DG-FFA100) and acetyl-CoA Assay Kit (Biovision; K317-100), respectively according to the manufacturer's instructions.

TUNEL assay. TUNEL assay was performed using In Situ Cell Death Detection Kit (Roche) on the deparaffinized cartilage section according to the manufacturer's instructions. Nuclear was stained with 4',6-diamidino-2-phenylindole, Dihydrochloride (DAPI). Fluorescence images were acquired using fluorescent microscopy.

Neutral lipid staining. Fixed cells or cryosection of human and mouse cartilages were stained using BODIPY ${ }^{493 / 503}$ (Thermo Fisher) for $20 \mathrm{~min}$ and mounted with
DAPI mounting medium (Vector Laboratories). Fluorescence images were acquired with cartilage images were acquired by EVOS FL Auto software v.1.7.

Lipid peroxidation staining. Live cells were stained using BODIPY581/591 (Invitrogen) probe followed by manufacturer's recommendation, and fluorescence intensity was quantified by image J $1.51 \mathrm{j} 8$.

Cell apoptosis assay. Cells were harvested and incubated with annexin $\mathrm{V}$ and propidium iodide (PI) solution from Muse Annexin V \& Dead Cell Kit (Luminex) according to the manufacturer's instructions. The fluorescence of annexin V and PI were analyzed by Muse Cell Analyzer (Merk Millipore).

Western blot. ACOT12 KO efficiency was confirmed by western blot using mouse liver. Mouse liver protein were extracted using RIPA buffer (Cell Signaling) with $2 \mathrm{mM}$ PMSF. Thirty micrograms of protein was separated by $10 \%$ polyacrylamide gel electrophoresis containing $0.1 \%$ SDS and transferred to nitrocellulose membranes (GE Healthcare). The membranes were incubated for $1 \mathrm{~h}$ at RT in TBS-T with $5 \%$ skim milk and probed with the following primary antibodies: ACOT12 (1:1000 dilution, Mybiosource; \#MBS273137), GAPDH (1:10,000 dilution, Bioworld; \#AP0066). The blots were developed with a HRP-conjugated anti-rabbit secondary antibody (1:2000 dilution, Bethyl Laboratories) and visualized with SuperSignal West Pico PLUS Chemiluminescent Substrate (Thermo Fisher).

Immunohistochemistry. For immunohistochemistry, antigen retrieval was performed with deparaffinized-rehydrated cartilage sections using $0.01 \mathrm{M}$ sodium citrate buffer $(0.05 \%$ Tween $20, \mathrm{pH} 6.0)$. Non-specific antibody-binding sites were blocked by incubation in $2.5 \%$ normal horse serum from ImmPRESS Universal Antibody Kit (Vector Laboratories). Sections were incubated overnight with the followed primary antibodies: ACOT12 (1:200 dilution, Mybiosource; \#MBS273137,), ADAMTS4 (1:100 dilution, Abcam, \#ab28285), FASN (1:100 dilution, Cell Signaling; \#3180,), HA-tag (1:100 dilution, Cell Signaling; \#3724), MMP13 (1:200 dilution, BioVision; \#3533), PPARa (1:100 dilution, Abcam; \#ab8934), and SCD1 (1:100 dilution, Abcam; \#ab19862). After incubation with peroxidase-conjugated secondary antibody for $30 \mathrm{~min}$, antigen was detected with ImmPACT DAB Substrate (Vector Laboratories). Hematoxylin or methyl-green was used to counterstain.

Quantitative real-time (qRT)-PCR. Total RNA was isolated from liver tissue using RNAiso Plus (TaKaRa) according to the manufacturer's instructions. Then, $1 \mu \mathrm{g}$ RNA was reverse transcribed using the $5 \times$ All-In-One RT Master Mix (ABM) Real-time PCR was performed with ABI StepOnePlus instrument (Applied Biosystems) using AMPIGENE qPCR Green Mix Hi-ROX (Enzo). The cycling protocol was 40 cycles of $95^{\circ} \mathrm{C}$ for $10 \mathrm{~s}, 57^{\circ} \mathrm{C}$ for $15 \mathrm{~s}$, and $72^{\circ} \mathrm{C}$ for $10 \mathrm{~s}$. All qRT-PCR reactions were performed in triplicate. The relative expression level of each gene was normalized to $18 \mathrm{~S}$ rRNA expression level. Forward and reverse primer sequences were listed in Supplementary Tables S1 and S2 (used in Fig. 1f), S3 (used in Fig. 3a).

\section{Synthesis of fluorescein isothiocyanate (FITC)-labeled chitosan (chitosan-}

FITC). Chitosan (50 mg, $267.4 \mu \mathrm{mol}$ of NH2) was dissolved in 1\% (v/v) acetic acid solution $(2.5 \mathrm{~mL})$. FITC $(10.4 \mathrm{mg}, 26.7 \mu \mathrm{mol})$ in $0.5 \mathrm{ml}$ methanol was slowly added onto the chitosan and reacted for $4 \mathrm{~h}$ in the dark. The obtained product was purified by dialysis (MWCO: $12-14 \mathrm{kDa}$, SpectraPor, USA) against an $\mathrm{HCl}$ solution (pH 5) for 2 days, followed by distilled and deionized water (DDW) for 2 days under darkness. The final product was lyophilized and held in the dark.

Preparation of chitosan-FITC/acetyl-CoA complexes. A chitosan-FITC stock solution was prepared by dissolving chitosan-FITC $(1 \mathrm{mg})$ in $0.05 \mathrm{~N} \mathrm{HCl}$ solutions $(1 \mathrm{ml})$. A reaction solution was prepared by making a 1:10 dilution of stock solution with pH 7.4 PBS. Ten microliters of acetyl-CoA $(50 \mathrm{mM})$ were added into the reaction solution and reacted for $6 \mathrm{~h}$ to ensure the formation of chitosan-FITC/ acetyl-CoA complexes. The final product was purified by dialysis (MWCO $12-14 \mathrm{kDa}$, SpectraPor, USA) against the DDW for 1 day.

Ethical approval. All animal studies were approved by the Wonkwang University Animal Care and Use Committee (\#WKU18-23, WKU19-09, WKU20-61) and were in compliance with the institutional guidelines. Human cartilage tissue collection was approved by the Human Subjects Committee of Wonkwang University Hospital (WKUH 201605-HRBR-041) and studies were performed in compliance with the institutional guidelines. Written informed consent was obtained from all adult patients or at least one guardian of each patient prior to the start of the experiment.

Statistical analyses. Results are expressed as the mean \pm SD. The mean values for RNA level and biochemical data of two groups were compared by unpaired twotailed Student's $t$ test. For more than two groups, one- or two-way ANOVA for multiple comparisons were used. Statistical tests with $P<0.05$ was considered 
significant and significance was defined as $* P<0.05,{ }^{* *} P<0.01$, ${ }^{* * *} P<0.001$, and $* * * * P<0.0001$. All statistical tests were performed with the software GraphPad Prism 6 (GraphPad).

Reporting summary. Further information on research design is available in the Nature Research Reporting Summary linked to this article.

\section{Data availability}

Microarray data of Human OA chondrocytes (GSE16464, GSE64394), primary mouse articular chondrocytes (IL-1 $\beta$-treated; GSE104793, HIF-2a-overexpressed; GSE104794, ZIP8-overexpressed; GSE104795) for Fig. 1g and OA-induced rat articular chondrocytes (GSE8077) for Fig. 3f were retrieved from GEO database. GSE data was analyzed by QIAGEN Ingeunity Pathway Analysis (IPA) software. Kyoto Encyclopedia of Genes and Genomes (KEGG) pathway analysis was performed by GSEA 3.0. Source data are provided with this paper.

\section{Code availability}

No unpublished code was used in this manuscript.

Received: 15 February 2019; Accepted: 3 December 2021; Published online: 05 January 2022

\section{References}

1. Buckwalter, J. A. \& Mankin, H. J. Articular cartilage: degeneration and osteoarthritis, repair, regeneration, and transplantation. Instruct. Course Lectures 47, 487-504 (1998).

2. Martel-Pelletier, J. et al. Osteoarthritis. Nat. Rev. Dis. Prim. 2, 16072 (2016).

3. Hashimoto, S., Takahashi, K., Amiel, D., Coutts, R. D. \& Lotz, M. Chondrocyte apoptosis and nitric oxide production during experimentally induced osteoarthritis. Arthritis Rheum. 41, 1266-1274 (1998).

4. Aizawa, T., Kon, T., Einhorn, T. A. \& Gerstenfeld, L. C. Induction of apoptosis in chondrocytes by tumor necrosis factor-alpha. J. Orthop. Res. 19, 785-796 (2001).

5. Vincenti, M. P. \& Brinckerhoff, C. E. Early response genes induced in chondrocytes stimulated with the inflammatory cytokine interleukin-1beta. Arthritis Res. 3, 381-388 (2001).

6. Yasuhara, R. et al. Interleukin-1beta induces death in chondrocyte-like ATDC5 cells through mitochondrial dysfunction and energy depletion in a reactive nitrogen and oxygen species-dependent manner. Biochemical J. 389, 315-323 (2005).

7. Mobasheri, A. et al. The role of metabolism in the pathogenesis of osteoarthritis. Nat. Rev. Rheumatol. 13, 302-311 (2017).

8. Zhuo, Q., Yang, W., Chen, J. \& Wang, Y. Metabolic syndrome meets osteoarthritis. Nat. Rev. Rheumatol. 8, 729-737 (2012).

9. Gkretsi, V., Simopoulou, T. \& Tsezou, A. Lipid metabolism and osteoarthritis: lessons from atherosclerosis. Prog. Lipid Res. 50, 133-140 (2011).

10. Cimmino, M. A. \& Cutolo, M. Plasma glucose concentration in symptomatic osteoarthritis: a clinical and epidemiological survey. Clin. Exp. Rheumatol. 8, 251-257 (1990).

11. Yusuf, E. et al. Association between weight or body mass index and hand osteoarthritis: a systematic review. Ann. Rheum. Dis. 69, 761-765 (2010).

12. Louati, K., Vidal, C., Berenbaum, F. \& Sellam, J. Association between diabetes mellitus and osteoarthritis: systematic literature review and meta-analysis. RMD Open 1, e000077 (2015).

13. Bonner, W. M., Jonsson, H., Malanos, C. \& Bryant, M. Changes in the lipids of human articular cartilage with age. Arthritis Rheum. 18, 461-473 (1975).

14. Lippiello, L., Walsh, T. \& Fienhold, M. The association of lipid abnormalities with tissue pathology in human osteoarthritic articular cartilage. Metab.: Clin. Exp. 40, 571-576 (1991).

15. Rocha, B., Ruiz-Romero, C. \& Blanco, F. J. Mass spectrometry imaging: a novel technology in rheumatology. Nat. Rev. Rheumatol. 13, 52-63 (2017).

16. Collins, D. H., Ghadially, F. N. \& Meachim, G. Intra-cellular lipids of cartilage. Ann. Rheum. Dis. 24, 123-135 (1965).

17. Villalvilla, A., Gomez, R., Largo, R. \& Herrero-Beaumont, G. Lipid transport and metabolism in healthy and osteoarthritic cartilage. Int. J. Mol. Sci. 14, 20793-20808 (2013).

18. Alvarez-Garcia, O., Rogers, N. H., Smith, R. G. \& Lotz, M. K. Palmitate has proapoptotic and proinflammatory effects on articular cartilage and synergizes with interleukin-1. Arthritis Rheumatol. 66, 1779-1788 (2014).

19. $\mathrm{Wu}, \mathrm{C}$. $\mathrm{L}$. et al. Dietary fatty acid content regulates wound repair and the pathogenesis of osteoarthritis following joint injury. Ann. Rheum. Dis. 74, 2076-2083 (2015).
20. Sekar, S. et al. Saturated fatty acids induce development of both metabolic syndrome and osteoarthritis in rats. Sci. Rep. 7, 46457 (2017).

21. Nazli, S. A., Loeser, R. F., Chubinskaya, S., Willey, J. S. \& Yammani, R. R. High fat-diet and saturated fatty acid palmitate inhibits IGF-1 function in chondrocytes. Osteoarthr. Cartil. 25, 1516-1521 (2017).

22. Song, J., Kang, Y. H., Yoon, S., Chun, C. H. \& Jin, E. J. HIF1alpha:CRAT:miR-144-3p axis dysregulation promotes osteoarthritis chondrocyte apoptosis and VLCFA accumulation. Oncotarget 8, 69351-69361 (2017).

23. Hart, D. J., Doyle, D. V. \& Spector, T. D. Association between metabolic factors and knee osteoarthritis in women: the Chingford Study. J. Rheumatol. 22, 1118-1123 (1995).

24. Knebel, B. et al. Fatty liver due to increased de novo lipogenesis: alterations in the hepatic peroxisomal proteome. Front. Cell Dev. Biol. 7, 248 (2019).

25. Ipsen, D. H., Lykkesfeldt, J. \& Tveden-Nyborg, P. Molecular mechanisms of hepatic lipid accumulation in non-alcoholic fatty liver disease. Cell. Mol. life Sci.: CMLS 75, 3313-3327 (2018).

26. Sanders, F. W. \& Griffin, J. L. De novo lipogenesis in the liver in health and disease: more than just a shunting yard for glucose. Biol. Rev. Camb. Philos. Soc. 91, 452-468 (2016).

27. Paglialunga, S. \& Dehn, C. A. Clinical assessment of hepatic de novo lipogenesis in non-alcoholic fatty liver disease. Lipids Health Dis. 15, 159 (2016).

28. Tillander, V., Alexson, S. E. H. \& Cohen, D. E. Deactivating fatty acids: acylCoA thioesterase-mediated control of lipid metabolism. Trends Endocrinol. Metab.: TEM 28, 473-484 (2017).

29. Hunt, M. C., Solaas, K., Kase, B. F. \& Alexson, S. E. Characterization of an acyl-coA thioesterase that functions as a major regulator of peroxisomal lipid metabolism. J. Biol. Chem. 277, 1128-1138 (2002).

30. Hunt, M. C. et al. A revised nomenclature for mammalian acyl-CoA thioesterases/hydrolases. J. Lipid Res. 46, 2029-2032 (2005).

31. Hunt, M. C. \& Alexson, S. E. The role acyl-CoA thioesterases play in mediating intracellular lipid metabolism. Prog. Lipid Res. 41, 99-130 (2002)

32. Hunt, M. C., Tillander, V. \& Alexson, S. E. Regulation of peroxisomal lipid metabolism: the role of acyl-CoA and coenzyme A metabolizing enzymes. Biochimie 98, 45-55 (2014).

33. Horibata, Y., Ando, H., Itoh, M. \& Sugimoto, H. Enzymatic and transcriptional regulation of the cytoplasmic acetyl-CoA hydrolase ACOT12. J. Lipid Res. 54, 2049-2059 (2013).

34. Waldstein, W. et al. OARSI osteoarthritis cartilage histopathology assessment system: a biomechanical evaluation in the human knee. J. Orthop. Res. 34, 135-140 (2016).

35. Pritzker, K. P. et al. Osteoarthritis cartilage histopathology: grading and staging. Osteoarthr. Cartil. 14, 13-29 (2006).

36. Glasson, S. S., Blanchet, T. J. \& Morris, E. A. The surgical destabilization of the medial meniscus (DMM) model of osteoarthritis in the $129 / \mathrm{SvEv}$ mouse. Osteoarthr. Cartil. 15, 1061-1069 (2007).

37. Maneiro, E. et al. Mitochondrial respiratory activity is altered in osteoarthritic human articular chondrocytes. Arthritis Rheum. 48, 700-708 (2003).

38. Yang, X. et al. Pyruvate kinase M2 modulates the glycolysis of chondrocyte and extracellular matrix in osteoarthritis. DNA Cell Biol. 37, 271-277 (2018).

39. Pietrocola, F., Galluzzi, L., Bravo-San Pedro, J. M., Madeo, F. \& Kroemer, G. Acetyl coenzyme A: a central metabolite and second messenger. Cell Metab. 21, 805-821 (2015).

40. Sivanand, S., Viney, I. \& Wellen, K. E. Spatiotemporal Control of Acetyl-CoA Metabolism in Chromatin Regulation. Trends Biochem. Sci. 43, 61-74 (2018).

41. Thakkar, H., Sharma, R. K., Mishra, A. K., Chuttani, K. \& Murthy, R. S. Efficacy of chitosan microspheres for controlled intra-articular delivery of celecoxib in inflamed joints. J. Pharm. Pharmacol. 56, 1091-1099 (2004).

42. Bernkop-Schnurch, A. \& Dunnhaupt, S. Chitosan-based drug delivery systems. Eur. J. Pharmaceutics Biopharmaceutics 81, 463-469 (2012).

43. Courties, A., Gualillo, O., Berenbaum, F. \& Sellam, J. Metabolic stress-induced joint inflammation and osteoarthritis. Osteoarthr. Cartil. 23, 1955-1965 (2015).

44. Loef, M., Schoones, J. W., Kloppenburg, M. \& Ioan-Facsinay, A. Fatty acids and osteoarthritis: different types, different effects. Jt. Bone Spine 86, 451-458 (2019).

45. Afif, H. et al. Peroxisome proliferator-activated receptor gammal expression is diminished in human osteoarthritic cartilage and is downregulated by interleukin-1beta in articular chondrocytes. Arthritis Res. Ther. 9, R31 (2007).

46. Clockaerts, S. et al. Peroxisome proliferator activated receptor alpha activation decreases inflammatory and destructive responses in osteoarthritic cartilage. Osteoarthr. Cartil. 19, 895-902 (2011).

47. Zhou, Y., Chen, X., Qu, N., Zhang, B. \& Xia, C. Chondroprotection of PPARalpha activation by WY14643 via autophagy involving Akt and ERK in LPS-treated mouse chondrocytes and osteoarthritis model. J. Cell. Mol. Med. 23, 2782-2793 (2019). 
48. Jutila, A. A. et al. Candidate mediators of chondrocyte mechanotransduction via targeted and untargeted metabolomic measurements. Arch. Biochem. Biophysics 545, 116-123 (2014).

49. Salinas, D. et al. Combining targeted metabolomic data with a model of glucose metabolism: toward progress in chondrocyte mechanotransduction. PLOS ONE 12, e0168326 (2017).

50. Zignego, D. L., Hilmer, J. K. \& June, R. K. Mechanotransduction in primary human osteoarthritic chondrocytes is mediated by metabolism of energy, lipids, and amino acids. J. Biomech. 48, 4253-4261 (2015).

51. Choudhary, C., Weinert, B. T., Nishida, Y., Verdin, E. \& Mann, M. The growing landscape of lysine acetylation links metabolism and cell signalling. Nat. Rev. Mol. Cell Biol. 15, 536-550 (2014).

52. Suematsu, N. \& Isohashi, F. Molecular cloning and functional expression of human cytosolic acetyl-CoA hydrolase. Acta Biochimica Polonica 53, 553-561 (2006).

53. Suematsu, N., Okamoto, K., Shibata, K., Nakanishi, Y. \& Isohashi, F. Molecular cloning and functional expression of rat liver cytosolic acetyl-CoA hydrolase. Eur. J. Biochem. 268, 2700-2709 (2001).

54. Wellen, K. E. et al. ATP-citrate lyase links cellular metabolism to histone acetylation. Science 324, 1076-1080 (2009).

55. Zhao, S. et al. ATP-citrate lyase controls a glucose-to-acetate metabolic switch. Cell Rep. 17, 1037-1052 (2016).

56. Ebisuno, S., Isohashi, F., Nakanishi, Y. \& Sakamoto, Y. Acetyl-CoA hydrolase: relation between activity and cholesterol metabolism in rat. Am. J. Physiol. 255, R724-R730 (1988).

57. Westin, M. A., Hunt, M. C. \& Alexson, S. E. Short- and medium-chain carnitine acyltransferases and acyl-CoA thioesterases in mouse provide complementary systems for transport of beta-oxidation products out of peroxisomes. Cell. Mol. Life Sci. 65, 982-990 (2008).

58. Chen, L. Y., Lotz, M., Terkeltaub, R. \& Liu-Bryan, R. Modulation of matrix metabolism by ATP-citrate lyase in articular chondrocytes. J. Biol. Chem. 293, 12259-12270 (2018)

59. Ameer, F., Scandiuzzi, L., Hasnain, S., Kalbacher, H. \& Zaidi, N. De novo lipogenesis in health and disease. Metab.: Clin. Exp. 63, 895-902 (2014).

\section{Acknowledgements}

This work was supported by the National Research Foundation of Korea (NRF) grant funded by the Korea government (MSIP) [2019R1A2C2002157 and 2021R1I1A3041149].

This paper is based on SJ Park's doctoral dissertation.

\section{Author contributions}

E.-J.J. and S.J.P. were designed the experiments, analyzed the data, and wrote the paper S.J.P. performed the experiments. C.C. provided human cartilage samples, I.-J.B. generated Acot12 KO mice, and J.-H.R. developed the chitosan/acetyl-CoA delivery system.

\section{Competing interests}

The authors declare no competing interests.

\section{Additional information}

Supplementary information The online version contains supplementary material available at https://doi.org/10.1038/s41467-021-27738-y.

Correspondence and requests for materials should be addressed to Eun-Jung Jin.

Peer review information Nature Communications thanks Francisco Blanco, Mohit Kapoor and the other, anonymous, reviewer(s) for their contribution to the peer review of this work.

Reprints and permission information is available at http://www.nature.com/reprints

Publisher's note Springer Nature remains neutral with regard to jurisdictional claims in published maps and institutional affiliations.

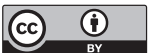

Open Access This article is licensed under a Creative Commons Attribution 4.0 International License, which permits use, sharing, adaptation, distribution and reproduction in any medium or format, as long as you give appropriate credit to the original author(s) and the source, provide a link to the Creative Commons license, and indicate if changes were made. The images or other third party material in this article are included in the article's Creative Commons license, unless indicated otherwise in a credit line to the material. If material is not included in the article's Creative Commons license and your intended use is not permitted by statutory regulation or exceeds the permitted use, you will need to obtain permission directly from the copyright holder. To view a copy of this license, visit http://creativecommons.org/ licenses/by/4.0/.

(C) The Author(s) 2022 